\title{
Dynamical Analysis of an SEIRS Model With Incomplete Recovery and Relapse on Networks and Its Optimal Vaccination Control
}

\section{Lei Zhang}

North University of China

Maoxing Liu ( $\square$ liumaoxing@126.com )

North University of China https://orcid.org/0000-0002-3342-7209

Qiang Hou

North University of China

Boli Xie

North University of China

\section{Research Article}

Keywords: networks, incomplete recovery, relapse, vaccination, global stability, optimal control

Posted Date: May 25th, 2021

DOl: https://doi.org/10.21203/rs.3.rs-510785/v1

License: (a) (i) This work is licensed under a Creative Commons Attribution 4.0 International License. Read Full License 


\title{
Dynamical analysis of an SEIRS model with incomplete recovery and relapse on networks and its optimal vaccination control $^{1}$
}

\author{
Lei Zhang ${ }^{a, b}$, Maoxing Liu ${ }^{a, b \dagger}$, Qiang $\mathrm{Hou}^{b}$, Boli Xie ${ }^{b}$ \\ a. School of Big Data, North University of China \\ Taiyuan, Shanxi, P. R. China, 030051 \\ b. Department of Mathematics, North University of China \\ Taiyuan, Shanxi, P. R. China, 030051
}

\begin{abstract}
For some infectious diseases, such as herpes and tuberculosis, there is incomplete recovery and relapse. These phenomena make them difficult to control. In consequence of this status, an SEIRS epidemic model with incomplete recovery and relapse on networks is established and the global dynamics is studied. The results show that when the basic reproduction number $R_{0} \leq 1$, the disease-free equilibrium is globally asymptotically stable; when $R_{0}>1$, the endemic equilibrium is globally asymptotically stable. In addition, in consideration of vaccination control strategy, an SVEIRS model is introduced and the optimal control is solved. At last, the theoretical results are illustrated with numerical simulations.
\end{abstract}

Keywords. networks; incomplete recovery; relapse; vaccination; global stability; optimal control

\section{Introduction}

In conventional mathematical models of epidemic, the population is usually divided into several compartments, which may include $S$ (susceptible), $E$ (infected but not fell ill and not infective, and also called exposed), $I$ (fell ill and infective), $R$ (recovered and immune). A susceptible can be infected by an infective individual and be fitted into either $E$ if it is not infective yet or $I$ if it is infective immediately. An exposed individual can be fitted into $I$ when it is infective and fell ill. An infective individual can be fitted into $R$ if it recovers and it is immune for the disease, or be fitted into $S$ if

\footnotetext{
${ }^{1}$ Research project is supported by the National Natural Science Foundation of China (Grant Nos. 12071445), Fund for Shanxi 1331KIRT, Graduate Innovation Project in Shanxi Province (2020BY104).

${ }^{\dagger}$ Corresponding author, E-mail: liumaoxing@126.com.
} 
it recovers and it is not immune. A recovered individual can also be fitted into $S$ when it lost immunity for the disease. For some diseases, there is incomplete recovery, and when incomplete recovery occurs, an infective individual can be fitted into $E$ again. Moreover, relapse may occur, that is, a recovered individual can be fitted back into $E$ for reactivation of the bacteria. Herpes [1] and tuberculosis $[2,3]$ are of this type.

Researches on incomplete recovery and relapse have been done for several decades. Tudor established an SIRI model for herpes and analysed the local stability of the equilibria [1]. Moreira and Wang extended the model and analyzed the global dynamical behaviour [4]. Feng et. al formulated one-strain and two-strain SLIT models of tuberculosis with incomplete treatment and relapse, and analyzed the global dynamical behaviour [5]. As an example, the basic reproduction number of the onestrain model was calculated by van den Driessche and Watmough [6]. According to herpes, van den Driessche and Zou analyzed a general relapse phenomenon by an infectious disease model (SIRI model) of integro-differential equations and gave the global asymptotical behavior of the model [7]. According to tuberculosis, van den Driessche et. al proposed a general mathematical model (SEIRI model) with latent and relapse and gave the global asymptotical behavior [8]. Based on the model of van den Driessche et. al [8], Liu et. al introduced nonlinear incidence of infection and analyzed the local asymptotical stability of the disease-free equilibrium, existence of the endemic equilibrium and the persistence of infection [9]. Georgescu and Zhang introduced nonlinear incidence of infection, recovery and relapse to an SIRI model and analyzed the global behavior [10]. Chen et. al proposed an SIRIS model with relapse and nonlinear incidence and analyzed the global behavior [11].

The researches above have a common assumption: homogeneous host and homogeneous contact. Taking the heterogeneous host into account, Feng et. al [12], Wang and Shu [13] studied multi-group epidemic models with latency and relapse, respectively. Pastor and Vespignani introduced contact heterogeneity into a traditional SIS model by scale-free networks for the first time [14]. In the past two decades, there have been a lot of significant works of epidemic models on networks [15]. But as far as we know, results of incomplete recovery and relapse on networks are scarce. Huo et. al introduced an SIRS model with relapse on a scale-free network, analyzed the 
global behavior, studied proportional immunization and targeted immunization [16]. Lahrouz et. al studied the global dynamics of an SIRI model with relapse on networks [17].

The purpose of studying infectious disease models is to control the spread of infectious diseases. As an infection control precaution, vaccination has been introduced into epidemic models for a long time $[18,19]$. Vaccination is a method widely used in the world which can prevent infection by improving the immunity of individuals. The advantage is that it can reduce infection and the pressure on the health system. For an individual, vaccination will not affect the normal life and work. Moreover the cost of vaccination is much lower than the cost of treatment after infection. However, vaccination and treatment take different ways. The target of treatment is the infected individuals. The target of vaccination is uncertain. The number of individuals which need vaccination is also uncertain. So an optimal vaccination strategy is required to achieve these goals: the least vaccination number, the least cost and effective control of disease spreading.

The method of solving the optimal control above is provided by the optimal control theory $[20,21]$. Using the optimal control theory, optimal vaccination control strategy has been studied for a long time [22-24]. Chen and Sun established and solved an optimal control problem of vaccination about SIRS model on networks [25]. Following [25], they added medication to the optimal vaccination control and solved the new problem [26].

On the basis of previous works, with the introduction of latency period, an SEIRS model with incomplete recovery and relapse on networks is established in Sect. 2. The global dynamics and the optimal vaccination control are studied. In particular, in Sect. 3, the global dynamics is studied. In Sect. 4, the optimal vaccination control is solved. In Sect. 5, numerical simulations is performed for sensitivity analysis and to support the theoretical results. In Sect. 6, the paper ends with discussion. 


\section{Model formulation}

We propose and study an SEIRS model with incomplete recovery and relapse on scale-free networks:

$$
\left\{\begin{array}{l}
\frac{d S_{k}(t)}{d t}=b\left(S_{k}(t)+E_{k}(t)+I_{k}(t)+R_{k}(t)\right)-\beta k S_{k}(t) \theta(t)+\delta_{1} R_{k}(t)-d S_{k}(t) \\
\frac{d E_{k}(t)}{d t}=\beta k S_{k}(t) \theta(t)+\gamma_{1} I_{k}(t)+\delta_{2} R_{k}(t)-(\alpha+d) E_{k}(t) \\
\frac{d I_{k}(t)}{d t}=\alpha E_{k}(t)-\left(\gamma_{1}+\gamma_{2}+d\right) I_{k}(t) \\
\frac{d R_{k}(t)}{d t}=\gamma_{2} I_{k}(t)-\left(\delta_{1}+\delta_{2}+d\right) R_{k}(t) .
\end{array}\right.
$$

In the process of disease transmission, there are four states: $S_{k}$ (susceptible), $E_{k}$ (exposed), $I_{k}$ (infected), $R_{k}$ (removed). Following [14], $P_{k}(k=1,2, \cdots, n)$ is the degree distribution, $\langle k\rangle=\sum_{k} k P_{k}$ is the average degree, and $\theta$ is the link probability pointing to an infected individual:

$$
\theta(t)=\frac{1}{\langle k\rangle} \sum_{k} k P_{k} I_{k}(t) .
$$

Assume that $b=d$, that is, the population of nodes with the same degree is constant and when one individual is removed from a node with degree $k$, another individual with degree $\mathrm{k}$ will be added. So $S_{k}(t)+E_{k}(t)+I_{k}(t)+R_{k}(t)=1$, and model (2.1) can be rewritten as:

$$
\left\{\begin{array}{l}
\frac{d S_{k}(t)}{d t}=b-\beta k S_{k}(t) \theta(t)+\delta_{1} R_{k}(t)-b S_{k}(t) \\
\frac{d E_{k}(t)}{d t}=\beta k S_{k}(t) \theta(t)+\gamma_{1} I_{k}(t)+\delta_{2} R_{k}(t)-(\alpha+b) E_{k}(t) \\
\frac{d I_{k}(t)}{d t}=\alpha E_{k}(t)-\left(\gamma_{1}+\gamma_{2}+b\right) I_{k}(t) \\
\frac{d R_{k}(t)}{d t}=\gamma_{2} I_{k}(t)-\left(\delta_{1}+\delta_{2}+b\right) R_{k}(t)
\end{array}\right.
$$

The transmission flow is shown in Fig. 1 and the biological meanings of the parameters are summarised in Table 1. From the biological background, all the parameters are positive constant and the initial condition for model (2.3) is in:

$$
\left\{\left(S_{k}(0), E_{k}(0), I_{k}(0), R_{k}(0)\right) \in R_{+}^{4 n}: S_{k}(0)+E_{k}(0)+I_{k}(0)+R_{k}(0)=1\right\}
$$




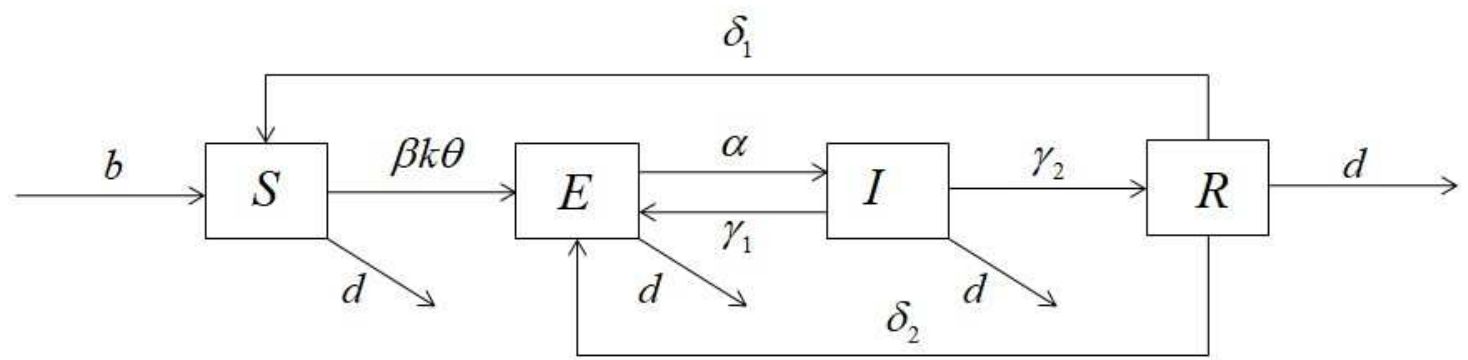

Figure 1: The disease transmission flow

\begin{tabular}{|c|c|c|c|}
\hline Parameter & Definition & \multicolumn{2}{|l|}{ Value } \\
\hline$b$ & Birth rate and death rate & \multicolumn{2}{|l|}{0.125} \\
\hline$\alpha$ & Transmission rate from $E$ to $I$ & \multicolumn{2}{|l|}{0.2} \\
\hline$\gamma_{1}$ & Transmission rate from $I$ to $E$ & \multicolumn{2}{|l|}{0.2} \\
\hline$\gamma_{2}$ & Transmission rate from $I$ to $R$ & \multicolumn{2}{|l|}{0.2} \\
\hline$\delta_{1}$ & Transmission rate from $R$ to $S$ & \multicolumn{2}{|l|}{0.2} \\
\hline$\delta_{2}$ & Transmission rate from $R$ to $E$ & \multicolumn{2}{|l|}{0.2} \\
\hline$w$ & Transmission rate from $V_{k}$ to $S$ & \multicolumn{2}{|l|}{0.2} \\
\hline \multirow{4}{*}{$\beta$} & \multirow{4}{*}{ Infection rate } & Fig. $3 \mathrm{a}$ & 0.02 \\
\hline & & Fig. $3 \mathrm{~b}$ & 0.034178 \\
\hline & & Fig. $2,3,5$ c, 6,7 & 0.1 \\
\hline & & Fig. $3 \mathrm{~d}$ & 0.15 \\
\hline \multirow{2}{*}{$B_{k}$} & \multirow{2}{*}{$\begin{array}{l}\text { Rate of the cost of immunization } \\
\text { to the cost of treatment }\end{array}$} & Fig. 6 & 0.05 \\
\hline & & Fig. 7 & 0.1 \\
\hline
\end{tabular}

Table 1: The definition and value of parameters in the model 


\section{Global behavior of the model}

In this section, we study the global dynamics of model (2.3). First, it is easily to have the following result:

Theorem 3.1. The set

$$
\Omega:=\left\{\left(S_{1}, E_{1}, I_{1}, R_{1}, \cdots, S_{n}, E_{n}, I_{n}, R_{n}\right) \in R_{+}^{4 n}: \text { forany }, S_{k}+E_{k}+I_{k}+R_{k}=1\right\}
$$

is a positively invariant set for model (2.3).

It is easy to find that $E_{0}=(1,0,0,0,1,0,0,0, \cdots, 1,0,0,0)$ is a disease-free equilibrium of model (2.3). Next we will study the basic reproduction number $R_{0}$ by the method of van den Driessche and Watmough [6], $R_{0}=\rho\left(F V^{-1}\right)$. Here $F_{3 n \times 3 n}$ is given by:

$$
F=\left(\begin{array}{cccc}
A_{11} & A_{12} & \cdots & A_{1 n} \\
A_{21} & A_{22} & \cdots & A_{2 n} \\
\vdots & \vdots & \ddots & \vdots \\
A_{n 1} & A_{n 2} & \cdots & A_{n n}
\end{array}\right)
$$

where

$$
A_{i j}=\left(\begin{array}{ccc}
0 & \frac{i j \beta P_{j}}{\langle k\rangle} & 0 \\
0 & 0 & 0 \\
0 & 0 & 0
\end{array}\right) .
$$

And $V_{3 n \times 3 n}=\operatorname{diag}\left(B_{1}, B_{2}, \cdots, B_{n}\right)$, where

$$
B_{k}=\left(\begin{array}{ccc}
b+\alpha & -\gamma_{1} & -\delta_{2} \\
-\alpha & b+\gamma_{1}+\gamma_{2} & 0 \\
0 & -\gamma_{2} & b+\delta_{1}+\delta_{2}
\end{array}\right) .
$$

Thus we have

$$
R_{0}=\rho\left(F V^{-1}\right)=\frac{\alpha \beta\left(b+\delta_{1}+\delta_{2}\right)}{b\left(b+\gamma_{1}+\gamma_{2}\right)\left(b+\delta_{1}+\delta_{2}\right)+\alpha\left(b^{2}+b \delta_{1}+b \delta_{2}+b \gamma_{2}+\delta_{1} \gamma_{2}\right)} \frac{\left\langle k^{2}\right\rangle}{\langle k\rangle} .
$$


Therefore, $R_{0}<1 \Leftrightarrow s(F-V)<0$ and $R_{0}>1 \Leftrightarrow s(F-V)>0$.

Remark 3.1. If the birth, death and incomplete recovery are not considered, that is, $b=\gamma_{1}=\delta_{2}=0$, then $R_{0}=\rho\left(F V^{-1}\right)=\frac{\beta}{\gamma_{2}} \frac{\left\langle k^{2}\right\rangle}{\langle k\rangle}$, which reduces to the result in Liu and Zhang [29].

Theorem 3.2. If $R_{0} \leq 1, E_{0}$ of model (2.3) is locally asymptotically stable; if $R_{0}>1, E_{0}$ is unstable.

Proof. From Theorem 2 of van den Driessche and Watmough [6], if $R_{0}<1, E_{0}$ is locally asymptotically stable; if $R_{0}>1, E_{0}$ is unstable. Next the case of $R_{0}=1$ will be proved. The Jacobian matrix $J_{4 n \times 4 n}$ is:

$$
J=\left(\begin{array}{cccc}
J_{11} & J_{12} & \cdots & J_{1 n} \\
J_{21} & J_{22} & \cdots & J_{2 n} \\
\vdots & \vdots & \ddots & \vdots \\
J_{n 1} & J_{n 2} & \cdots & J_{n n}
\end{array}\right)
$$

where

$$
J_{i i}=\left(\begin{array}{cccc}
-b & 0 & -\frac{i^{2} \beta P_{i}}{\langle k\rangle} & \delta_{1} \\
0 & -(b+\alpha) & \frac{i^{2} \beta P_{i}}{\langle k\rangle}+\gamma_{1} & \delta_{2} \\
0 & \alpha & -\left(b+\gamma_{1}+\gamma_{2}\right) & 0 \\
0 & 0 & \gamma_{2} & -\left(b+\delta_{1}+\delta_{2}\right)
\end{array}\right)
$$

and when $i \neq j$

$$
J_{i j}=\left(\begin{array}{cccc}
0 & 0 & -\frac{i j \beta P_{j}}{\langle k\rangle} & 0 \\
0 & 0 & \frac{i j \beta P_{j}}{\langle k\rangle} & 0 \\
0 & 0 & 0 & 0 \\
0 & 0 & 0 & 0
\end{array}\right)
$$

The characteristic equation of $J$ is

$$
f(\lambda)=\frac{1}{\langle k\rangle}(b+\lambda)^{n} f_{1}^{n-1}(\lambda) f_{2}(\lambda)
$$

where

$$
f_{1}(\lambda)=\lambda^{3}+C_{12} \lambda^{2}+C_{11} \lambda+C_{10},
$$




$$
f_{2}(\lambda)=\langle k\rangle \lambda^{3}+C_{22} \lambda^{2}+C_{21} \lambda+C_{20}
$$

and following [14], $\left\langle k^{2}\right\rangle=\sum_{k} k^{2} P_{k}$,

$$
\begin{aligned}
& C_{12}=3 b+\alpha+\delta_{1}+\delta_{2}+\gamma_{1}+\gamma_{2}, \\
& C_{11}=\left(2 b+\alpha+\gamma_{1}+\gamma_{2}\right)\left(2 b+\delta_{1}+\delta_{2}\right)+\alpha \gamma_{2}-b^{2}, \\
& C_{10}=b\left(b+\gamma_{1}+\gamma_{2}\right)\left(b+\delta_{1}+\delta_{2}\right)+\alpha\left(b^{2}+b \delta_{1}+b \delta_{2}+b \gamma_{2}+\delta_{1} \gamma_{2}\right), \\
& C_{22}=C_{12}\langle k\rangle, \\
& C_{21}=C_{11}\langle k\rangle-\alpha \beta\left\langle k^{2}\right\rangle, \\
& C_{20}=C_{10}\langle k\rangle-\alpha \beta\left(b+\delta_{1}+\delta_{2}\right)\left\langle k^{2}\right\rangle .
\end{aligned}
$$

Since $C_{12}, C_{11}, C_{10}>0$, and $C_{12} C_{11}>C_{10}$, by the Routh-Hurwitz criterion, all roots of $f_{1}(\lambda)=0$ have negative real parts. When $R_{0}=1, C_{20}=0, C_{22}, C_{21}>0$, one root of $f_{2}(\lambda)$ is zero and other roots have negative real parts. The proof is completed.

Theorem 3.3. If $R_{0} \leq 1, E_{0}$ of model (2.3) is globally asymptotically stable.

Proof. Define $V_{1}(t)=\frac{1}{\langle k\rangle} \sum_{k} k P_{k} \int_{S_{k}}^{1}(1-x) d x, V_{2}(t)=\frac{1}{\langle k\rangle} \sum_{k} k P_{k} E_{k}, V_{3}(t)=\frac{b+\alpha}{\alpha} \theta$, $V_{4}(t)=\frac{\delta_{2}}{\langle k\rangle\left(b+\delta_{1}+\delta_{2}\right)} \sum_{k} k P_{k} R_{k}, V(t)=V_{1}(t)+V_{2}(t)+V_{3}(t)+V_{4}(t)$. Then

$$
\begin{gathered}
\dot{V}_{1}=\frac{1}{\langle k\rangle} \sum_{k} k P_{k}\left(S_{k}-1\right) \dot{S}_{k} \\
=\frac{1}{\langle k\rangle} \sum_{k} k P_{k}\left(S_{k}-1\right)\left[-b\left(S_{k}-1\right)-\beta k \theta\left(S_{k}-1\right)-\beta k \theta+\delta_{1} R_{k}\right] \\
=\frac{1}{\langle k\rangle} \sum_{k} k P_{k}\left[-(b+\beta k \theta)\left(S_{k}-1\right)^{2}-\beta k \theta\left(S_{k}-1\right)+\delta_{1} R_{k}\left(S_{k}-1\right)\right] \\
\leq \frac{1}{\langle k\rangle} \sum_{k} k P_{k}\left[-(b+\beta k \theta)\left(S_{k}-1\right)^{2}-\beta k \theta\left(S_{k}-1\right)\right] \\
=-\frac{1}{\langle k\rangle} \sum_{k} k P_{k}(b+\beta k \theta)\left(S_{k}-1\right)^{2}+\frac{\beta \theta}{\langle k\rangle} \sum_{k} k^{2} P_{k}-\frac{\beta \theta}{\langle k\rangle} \sum_{k} k^{2} P_{k} S_{k}, \\
\dot{V}_{2}=\frac{1}{\langle k\rangle} \sum_{k} k P_{k} \dot{E}_{k} \\
=\frac{1}{\langle k\rangle} \sum_{k} k P_{k}\left[-(b+\alpha) E_{k}+\beta k \theta S_{k}+\gamma_{1} I_{k}+\delta_{2} R_{k}\right] \\
=-\frac{b+\alpha}{\langle k\rangle} \sum_{k} k P_{k} E_{k}+\frac{\beta \theta}{\langle k\rangle} \sum_{k} k^{2} P_{k} S_{k}+\gamma_{1} \theta+\frac{\delta_{2}}{\langle k\rangle} \sum_{k} k P_{k} R_{k}, \\
\dot{V}_{3}=\frac{b+\alpha}{\alpha} \frac{1}{\langle k\rangle} \sum_{k} k P_{k} \dot{I}_{k} \\
=\frac{b+\alpha}{\alpha} \frac{1}{\langle k\rangle} \sum_{k} k P_{k}\left[\alpha E_{k}-\left(\gamma_{1}+\gamma_{2}+b\right) I_{k}\right] \\
=\frac{b+\alpha}{\langle k\rangle} \sum_{k} k P_{k} E_{k}-\frac{b+\alpha}{\alpha}\left(\gamma_{1}+\gamma_{2}+b\right) \theta
\end{gathered}
$$


and

$$
\begin{aligned}
\dot{V}_{4} & =\frac{\delta_{2}}{\langle k\rangle\left(b+\delta_{1}+\delta_{2}\right)} \sum_{k} k P_{k} \dot{R}_{k} \\
& =\frac{\delta_{2}}{\langle k\rangle\left(b+\delta_{1}+\delta_{2}\right)} \sum_{k} k P_{k}\left[\gamma_{2} I_{k}-\left(\delta_{1}+\delta_{2}+b\right) R_{k}\right] \\
& =\frac{\gamma_{2} \delta_{2}}{b+\delta_{1}+\delta_{2}} \theta-\frac{\delta_{2}}{\langle k\rangle} \sum_{k} k P_{k} R_{k} .
\end{aligned}
$$

Therefore,

$$
\begin{aligned}
\dot{V}= & \dot{V}_{1}+\dot{V}_{2}+\dot{V}_{3}+\dot{V}_{4} \leq-\frac{1}{\langle k\rangle} \sum_{k} k P_{k}(b+\beta k \theta)\left(S_{k}-1\right)^{2} \\
& +\theta\left[\beta \frac{\left\langle k^{2}\right\rangle}{\langle k\rangle}+\gamma_{1}-\frac{b+\alpha}{\alpha}\left(\gamma_{1}+\gamma_{2}+b\right)+\frac{\gamma_{2} \delta_{2}}{b+\delta_{1}+\delta_{2}}\right] \\
= & -\frac{1}{\langle k\rangle} \sum_{k} k P_{k}(b+\beta k \theta)\left(S_{k}-1\right)^{2}+\theta\left[\gamma_{1}-\frac{b+\alpha}{\alpha}\left(\gamma_{1}+\gamma_{2}+b\right)+\frac{\gamma_{2} \delta_{2}}{b+\delta_{1}+\delta_{2}}\right]\left(1-R_{0}\right) .
\end{aligned}
$$

Note that $\gamma_{1}-\frac{b+\alpha}{\alpha}\left(\gamma_{1}+\gamma_{2}+b\right)+\frac{\gamma_{2} \delta_{2}}{b+\delta_{1}+\delta_{2}}<0$, then when $R_{0} \leq 1, \dot{V} \leq 0$ and $\dot{V}=0$ if and only if $S_{k}=1$ and $I_{k}=0$. By the LaSalle invariant principle [30], $\lim _{t \rightarrow \infty} S_{k}=1$ and $\lim _{t \rightarrow \infty} I_{k}=0$. By Theorem 3.1, $\lim _{t \rightarrow \infty} E_{k}=\lim _{t \rightarrow \infty} R_{k}=0$. Then $E_{0}$ is globally attractive, combining Theorem $3.2, E_{0}$ is globally asymptotically stable when $R_{0} \leq 1$.

Theorem 3.4. If $R_{0}>1$ there is a unique endemic equilibrium $E_{+}$of model (2.3).

Proof. Let model (2.3) be zero. We have

$$
\left\{\begin{array}{l}
b\left(S_{k}+E_{k}(t)+I_{k}+R_{k}\right)-\beta k S_{k} \theta(t)+\delta_{1} R_{k}-b S_{k}=0 \\
\beta k S_{k} \theta+\gamma_{1} I_{k}+\delta_{2} R_{k}-(\alpha+b) E_{k}=0 \\
\alpha E_{k}-\left(\gamma_{1}+\gamma_{2}+b\right) I_{k}=0 \\
\gamma_{2} I_{k}-\left(\delta_{1}+\delta_{2}+b\right) R_{k}=0 .
\end{array}\right.
$$

Solve Eq. (3.17), we have

$$
\begin{aligned}
S_{k}^{*} & =\frac{C_{10}}{C_{10}+k \beta\left[\left(b+\alpha+\gamma_{1}+\gamma_{2}\right)\left(b+\delta_{1}+\delta_{2}\right)+\alpha \delta_{2}\right] \theta}, \\
E_{k}^{*} & =\frac{k \beta\left(b+\gamma_{1}+\gamma_{2}\right)\left(b+\delta_{1}+\delta_{2}\right) \theta}{C_{10}+k \beta\left[\left(b+\alpha+\gamma_{1}+\gamma_{2}\right)\left(b+\delta_{1}+\delta_{2}\right)+\alpha \delta_{2}\right] \theta}, \\
I_{k}^{*} & =\frac{k \alpha \beta\left(b+\delta_{1}+\delta_{2}\right) \theta}{C_{10}+k \beta\left[\left(b+\alpha+\gamma_{1}+\gamma_{2}\right)\left(b+\delta_{1}+\delta_{2}\right)+\alpha \delta_{2}\right] \theta} \\
R_{k}^{*} & =\frac{k \alpha \beta \gamma_{2} \theta}{C_{10}+k \beta\left[\left(b+\alpha+\gamma_{1}+\gamma_{2}\right)\left(b+\delta_{1}+\delta_{2}\right)+\alpha \delta_{2}\right] \theta} .
\end{aligned}
$$


Substituting the third equation of Eq. (3.18) into Eq. (2.2), we have $g(\theta)-\theta=0$, where

$$
\begin{aligned}
& g(\theta)=\frac{1}{\langle k\rangle} \sum_{k} k^{2} P_{k} \frac{g_{1} \theta}{g_{2}+k g_{3} \theta}, \\
& g_{1}=\alpha \beta\left(b+\delta_{1}+\delta_{2}\right), \\
& g_{2}=C_{10}, \\
& g_{3}=\beta\left[\left(b+\alpha+\gamma_{1}+\gamma_{2}\right)\left(b+\delta_{1}+\delta_{2}\right)+\alpha \delta_{2}\right] .
\end{aligned}
$$

Set $G(\theta)=g(\theta)-\theta$, then $G(0)=0, G(1)<0, \dot{G}(0)=R_{0}-1, \dot{G}(1)<0, \ddot{G}(\theta)<0$. If $R_{0} \leq 1, \dot{G}(0) \leq 0, \ddot{G}(\theta)<0$, it follows that $\dot{G}(\theta)<0$ for $\theta \in(0,1)$ and here $G(\theta)=g(\theta)-\theta$ has no positive solutions. If $R_{0}>1$, then $\dot{G}(0)>0, \dot{G}(1)<0$, $\ddot{G}(\theta)<0$, thus $\dot{G}(\theta)=0$ has a unique positive solution $\theta_{1}$. In addition, when $0 \leq \theta \leq \theta_{1}, G(\theta)$ strictly increases, so $G\left(\theta_{1}\right)>0 ;$ when $\theta_{1} \leq \theta \leq 1, G(\theta)$ strictly decreases with $G(1)<0$. Therefore, $G(\theta)=g(\theta)-\theta$ has a unique positive solution.

Next, similar to [31], use Theorem 3.2 of Zhao and Jing [32], we can get the global asymptotical stability of $E_{+}$.

Theorem 3.5. If $R_{0}>1$, then $E_{+}$of model (2.3) is globally asymptotically stable.

Proof. For $S_{k}+E_{k}+I_{k}+R_{k}=1$, we just need to study the following model:

$$
\left\{\begin{array}{l}
\frac{d E_{k}}{d t}=\beta k S_{k} \theta+\gamma_{1} I_{k}+\delta_{2} R_{k}-(\alpha+b) E_{k} \\
\frac{d I_{k}}{d t}=\alpha E_{k}-\left(\gamma_{1}+\gamma_{2}+b\right) I_{k} \\
\frac{d R_{k}}{d t}=\gamma_{2} I_{k}-\left(\delta_{1}+\delta_{2}+b\right) R_{k} .
\end{array}\right.
$$

Let $X=\left\{E_{1}, I_{1}, R_{1}, \cdots, E_{n}, I_{n}, R_{n}\right\}, \frac{d E_{k}}{d t}=g_{k 1}, \frac{d I_{k}}{d t}=g_{k 2}, \frac{d R_{k}}{d t}=g_{k 3}, g=$ $\left(g_{11}, g_{12}, g_{13}, \cdots, g_{n 1}, g_{n 2}, g_{n 3}\right)$. Obviously $g$ is continuous. For any $x \in X, \frac{d g_{k 1}}{d x} \geq 0$ if $x_{3(k-1)+1} \neq E_{k}, \frac{d g_{k 2}}{d x} \geq 0$ if $x_{3(k-1)+2} \neq I_{k}, \frac{d g_{k 3}}{d x} \geq 0$ if $x_{3(k-1)+3} \neq R_{k}$, so $g$ is cooperative.

(1)Remove $S_{1}, S_{2}, \cdots, S_{n}$ of the elements in the set $\Omega$, reduce the dimension of $\Omega$ and denote it by $\Omega^{*}$. From Eq. (3.20), $g(0)=0$ and $g_{i j}(x) \geq 0$ for all $X \in \Omega^{*}$ with $x_{3(i-1)+j}=0, i=1,2, \cdots, n$.

(2)For every $x \in \Omega^{*}, D g(x)$ is irreducible. 
(3) Let $\varepsilon \in(0,1)$, for $k=1,2, \cdots, n$,

$$
\begin{aligned}
& g_{k 1}(\varepsilon x)=\varepsilon \beta k\left(1-\varepsilon E_{k}-\varepsilon I_{k}-\varepsilon R_{k}\right) \theta+\varepsilon \gamma_{1} I_{k}+\varepsilon \delta_{2} R_{k}-\varepsilon(\alpha+b) E_{k} \\
& >\varepsilon \beta k\left(1-E_{k}-I_{k}-R_{k}\right) \theta+\varepsilon \gamma_{1} I_{k}+\varepsilon \delta_{2} R_{k}-\varepsilon(\alpha+b) E_{k} \\
& =\varepsilon g_{k 1}(x) ; \\
& \quad g_{k 2}(\varepsilon x)=\alpha\left(1-\varepsilon S_{k}-\varepsilon I_{k}-\varepsilon R_{k}\right)-\varepsilon\left(\gamma_{1}+\gamma_{2}+b\right) I_{k} \\
& >\alpha\left(\varepsilon-\varepsilon S_{k}-\varepsilon I_{k}-\varepsilon R_{k}\right)-\varepsilon\left(\gamma_{1}+\gamma_{2}+b\right) I_{k} \\
& =\varepsilon g_{k 2}(x) ; \\
& \quad g_{k 3}(\varepsilon x)=\gamma_{2}\left(1-\varepsilon S_{k}-\varepsilon E_{k}-\varepsilon R_{k}\right)-\varepsilon\left(\delta_{1}+\delta_{2}+b\right) R_{k} \\
& >\gamma_{2}\left(\varepsilon-\varepsilon S_{k}-\varepsilon E_{k}-\varepsilon R_{k}\right)-\varepsilon\left(\delta_{1}+\delta_{2}+b\right) R_{k} \\
& =\varepsilon g_{k 3}(x) ;
\end{aligned}
$$

Then the right side of model (3.20) are strictly sub-linear on $\Omega^{*}$. Model (3.20) satisfies the condition of Theorem 3.2 of Zhao and Jing [32]. When $R_{0}>1, s(D g(0))=$ $s(F-V)>0$, by Theorem 3.1,3.2 and 3.4, $E_{+}$is globally asymptotically stable.

\section{Optimal vaccination control}

In order to save the vaccination control cost and ensure the achievement of the vaccination control goal, we will use the optimal control theory to establish and solve a time-varying vaccination control problem. Define the time-varying vaccination control variables $v(t)=\left(v_{1}(t), v_{2}(t), \cdots, v_{n}(t)\right)$, where $0 \leq v_{k}(t) \leq 1$ and $t \in[0, T]$, $T>0$ is the terminal time. Then the optimal vaccination control problem of model 
(2.3) can be written as

$$
\left\{\begin{array}{l}
\frac{d S_{k}(t)}{d t}=b-\beta k S_{k}(t) \theta(t)+\delta_{1} R_{k}(t)-b S_{k}(t)-v_{k}(t) S_{k}(t)+w V_{k}(t) \\
\frac{d E_{k}(t)}{d t}=\beta k S_{k}(t) \theta(t)+\gamma_{1} I_{k}(t)+\delta_{2} R_{k}(t)-(\alpha+b) E_{k}(t) \\
\frac{d I_{k}(t)}{d t}=\alpha E_{k}(t)-\left(\gamma_{1}+\gamma_{2}+b\right) I_{k}(t) \\
\frac{d R_{k}(t)}{d t}=\gamma_{2} I_{k}(t)-\left(\delta_{1}+\delta_{2}+b\right) R_{k}(t)+v_{k}(t) S_{k}(t) \\
\frac{d V_{k}(t)}{d t}=v_{k}(t) S_{k}(t)-w V_{k}(t)
\end{array}\right.
$$

where $V_{k}(t)$ is the density of people with immunity after vaccination at node $k, w$ is the rate of loss of immunity. The objective function is

$$
J(v)=\int_{0}^{T} L(v(t)) d t
$$

and $L(v(t))$ is the Lagrangian function:

$$
\sum_{k=1}^{n}\left[I_{k}(t)+\frac{1}{2} B_{k} v_{k}^{2}(t)\right]
$$

where $B_{k}$ is the ratio of the cost of immunization to the cost of treatment. Our goal is to find an time-varying vaccination control (optimal control) $v^{*}$ such that $J\left(v^{*}\right)=\min _{v(t) \in V} J(v)$, where $V=\left\{v(t), 0 \leq v_{k}(t), t \in[0, T]\right\}$. Next, use the same method as $[27,28]$, we obtain the existence of the optimal vaccination control of Eq. (4.1):

Theorem 4.1. There exists an optimal vaccination control $v^{*}(t)$.

Then we solve the optimal vaccination control problem by the Pontryagin's minimum Principle [21]. We define the Hamiltonian $H$ as follows:

$$
H=L(v)+\sum_{k=1}^{n}\left[\lambda_{1 k}(t) \dot{S}_{k}(t)+\lambda_{2 k}(t) \dot{E}_{k}(t)+\lambda_{3 k}(t) \dot{I}_{k}(t)+\lambda_{4 k}(t) \dot{R}_{k}(t)+\lambda_{5 k}(t) \dot{V}_{k}(t)\right]
$$

where $\lambda_{i k}(t),(k=1,2, \cdots, 5)$, are the adjoint variables. We denote $\left(S_{1}^{*}, E_{1}^{*}, I_{1}^{*}, R_{1}^{*}, V_{1}^{*}\right.$, $\left.\cdots, S_{n}^{*}, E_{n}^{*}, I_{n}^{*}, R_{n}^{*}, V_{n}^{*}\right)$ as $O_{k}^{*}$. We have the following theorem:

Theorem 4.2. Denote $v^{*}(t)$ as the optimal control, and $\theta^{*}(t)=\frac{1}{\langle k\rangle} \sum_{k} k P(k) I_{k}^{*}(t)$ is the link probability in the optimal state, if $O_{k}^{*}$ is the optimal solution of Eq. (4.1) 
with the condition

$$
\lambda_{i k}(T)=0, i=1,2, \cdots, 5 \quad \text { and } \quad k=1,2, \cdots, n .
$$

then $\lambda_{i k}(t),(k=1,2, \cdots, 5)$ satisfy:

$$
\left\{\begin{aligned}
\dot{\lambda}_{1 k}(t) & =\left(\beta k \theta^{*}(t)+b+v_{k}^{*}(t)\right) \lambda_{1 k}(t)-\beta k \theta^{*}(t) \lambda_{2 k}(t)-v_{k}^{*}(t) \lambda_{5 k}(t) \\
\dot{\lambda}_{2 k}(t) & =(\alpha+b) \lambda_{2 k}(t)-\alpha \lambda_{3 k}(t) \\
\dot{\lambda}_{3 k}(t) & =-1+\frac{\beta k P_{k}}{\langle k\rangle} \sum_{k=1}^{n} k S_{k}^{*}(t)\left(\lambda_{1 k}(t)-\lambda_{2 k}(t)\right)-\gamma_{1} \lambda_{2 k}(t) \\
& +\left(\gamma_{1}+\gamma_{2}+b\right) \lambda_{3 k}(t)-\gamma_{2} \lambda_{4 k}(t) \\
\dot{\lambda}_{4 k}(t) & =-\delta_{1} \lambda_{1 k}(t)-\delta_{2} \lambda_{2 k}(t)+\left(\delta_{1}+\delta_{2}+b\right) \lambda_{4 k}(t) \\
\dot{\lambda}_{5 k}(t) & =w\left(\lambda_{5 k}-\lambda_{1 k}\right)
\end{aligned}\right.
$$

And the optimal control can be obtained

$$
v_{k}^{*}(t)=\min \left\{\max \left(0, \frac{\left(\lambda_{1 k}(t)-\lambda_{5 k}(t)\right) S_{k}^{*}(t)}{B_{k}}\right), 1\right\} .
$$

Proof. Following [21], $\lambda_{i k}(t),(k=1,2, \cdots, 5)$ can be calculated by

$$
\begin{gathered}
\dot{\lambda}_{1 k}(t)=-\left.\frac{\partial H}{\partial S_{k}}\right|_{O_{k}^{*}(t)}, \dot{\lambda}_{2 k}(t)=-\left.\frac{\partial H}{\partial E_{k}}\right|_{O_{k}^{*}(t)}, \dot{\lambda}_{3 k}(t)=-\left.\frac{\partial H}{\partial I_{k}}\right|_{O_{k}^{*}(t)}, \\
\dot{\lambda}_{4 k}(t)=-\left.\frac{\partial H}{\partial R_{k}}\right|_{O_{k}^{*}(t)}, \dot{\lambda}_{5 k}(t)=-\left.\frac{\partial H}{\partial V_{k}}\right|_{O_{k}^{*}(t)} .
\end{gathered}
$$

With transversality condition (4.5) and

$$
\left.\frac{\partial H}{\partial v_{k}}\right|_{O_{k}^{*}(t)}=0,
$$

the adjoint varibles can be calculated by:

$$
\begin{gathered}
\dot{\lambda}_{1 k}(t)=-\left.\frac{\partial H}{\partial S_{k}}\right|_{O_{k}^{*}(t)} \\
=-\left[\left(-\beta k \theta^{*}(t)-b-v_{k}^{*}(t)\right) \lambda_{1 k}(t)+\beta k \theta^{*}(t) \lambda_{2 k}(t)+v_{k}^{*}(t) \lambda_{5 k}(t)\right] \\
=\left(\beta k \theta^{*}(t)+b+v_{k}^{*}(t)\right) \lambda_{1 k}(t)-\beta k \theta^{*}(t) \lambda_{2 k}(t)-v_{k}^{*}(t) \lambda_{5 k}(t), \\
\dot{\lambda}_{2 k}(t)=-\left.\frac{\partial H}{\partial E_{k}}\right|_{O_{k}^{*}(t)}=-\left[-(\alpha+b) \lambda_{2 k}(t)+\alpha \lambda_{3 k}(t)\right] \\
=(\alpha+b) \lambda_{2 k}(t)-\alpha \lambda_{3 k}(t),
\end{gathered}
$$




$$
\begin{aligned}
\dot{\lambda}_{3 k}(t)= & -\left.\frac{\partial H}{\partial I_{k}}\right|_{O_{k}^{*}(t)} \\
= & -\left[1+\frac{\beta k P_{k}}{\langle k\rangle} \sum_{k=1}^{n}\left(-k S_{k}^{*}(t)\right) \lambda_{1 k}(t)+\frac{\beta k P_{k}}{\langle k\rangle} \sum_{k=1}^{n} k S_{k}^{*}(t) \lambda_{2 k}(t)\right. \\
& \left.+\gamma_{1} \lambda_{2 k}(t)-\left(\gamma_{1}+\gamma_{2}+b\right) \lambda_{3 k}(t)+\gamma_{2} \lambda_{4 k}(t)\right] \\
= & -1+\frac{\beta k P_{k}}{\langle k\rangle} \sum_{k=1}^{n} k S_{k}^{*}(t)\left(\lambda_{1 k}(t)-\lambda_{2 k}(t)\right)-\gamma_{1} \lambda_{2 k}(t) \\
& +\left(\gamma_{1}+\gamma_{2}+b\right) \lambda_{3 k}(t)-\gamma_{2} \lambda_{4 k}(t), \\
\dot{\lambda}_{4 k}(t)= & -\left.\frac{\partial H}{\partial R_{k}}\right|_{O_{k}^{*}(t)}=-\left[\delta_{1} \lambda_{1 k}(t)+\delta_{2} \lambda_{2 k}(t)-\left(\delta_{1}+\delta_{2}+b\right) \lambda_{4 k}(t)\right] \\
= & -\delta_{1} \lambda_{1 k}(t)-\delta_{2} \lambda_{2 k}(t)+\left(\delta_{1}+\delta_{2}+b\right) \lambda_{4 k}(t), \\
\dot{\lambda}_{5 k}(t)= & -\left.\frac{\partial H}{\partial V_{k}}\right|_{O_{k}^{*}(t)}=-\left[w \lambda_{1 k}(t)-w \lambda_{5 k}(t)\right]=w\left[\lambda_{5 k}(t)-w \lambda_{1 k}(t)\right],
\end{aligned}
$$

By

$$
\left.\frac{\partial H}{\partial v_{k}}\right|_{O_{k}^{*}(t)}=B_{k} v_{k}^{*}(t)-\lambda_{1 k}(t) S_{k}^{*}(t)+\lambda_{5 k}(t) S_{k}^{*}(t)=0
$$

the optimal vaccination control is

$$
v_{k}^{*}(t)=\min \left\{\max \left(0, \frac{\left(\lambda_{1 k}(t)-\lambda_{5 k}(t)\right) S_{k}^{*}(t)}{B_{k}}\right), 1\right\}
$$

\section{$5 \quad$ Numerical simulations}

In this section, numerical simulations will be presented to show the correlation and sensitivity of the basic reproduction number to the parameters, to illustrate the global behavior of model 2.3 in Sect. 3, and to show the optimal control problem (4.1) in Sect. 4. Use 4 steps Runge Kutta algorithm, the differential equations are solved by Matlab. The dynamical process is considered on powerlaw networks with $P_{k}=c k^{-\gamma}$ and $\sum_{k=1}^{n} P_{k}=1,\langle k\rangle=\sum_{k=1}^{n} k P_{k}=1.3668,\left\langle k^{2}\right\rangle=\sum_{k=1}^{n} k^{2} P_{k}=5.6510$. For simulation, $\gamma=-3, n=500, T=30$, other parameters are listed in Table 1 .

Fig. 2 shows the sensitivity of the basic reproduction number $R_{0}$ to $\gamma_{1}, \gamma_{2}, \delta_{1}$ and $\delta_{2}$. $R_{0}$ decreases with the increase of $\gamma_{1}, \gamma_{2}, \delta_{1}$, and increases with the increase of $\delta_{2}$. The incomplete recovery is related to $\gamma_{1}$ and the relapse is related to $\delta_{2}$. Only relapse 


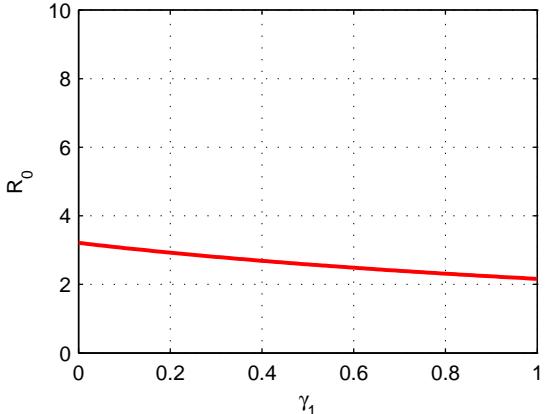

(a) $R_{0}$ as a function of $\gamma_{1}$

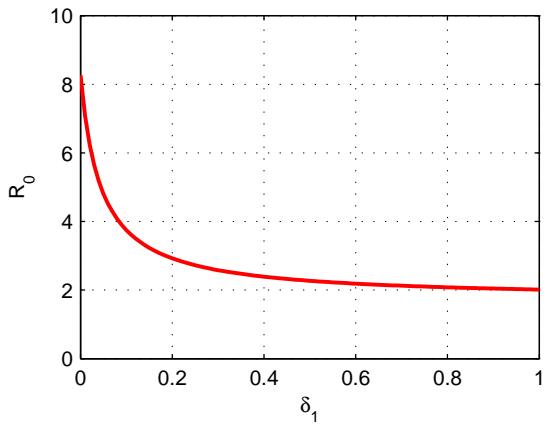

(c) $R_{0}$ as a function of $\delta_{1}$

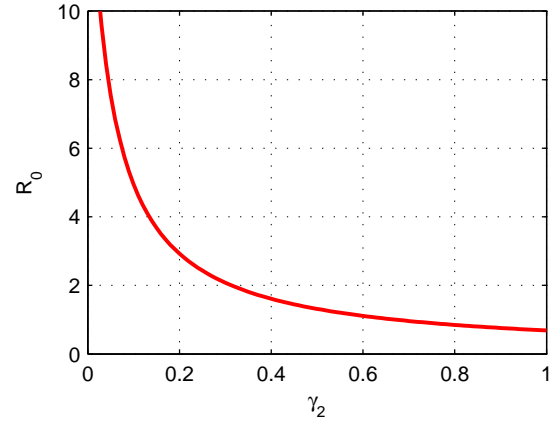

(b) $R_{0}$ as a function of $\gamma_{2}$

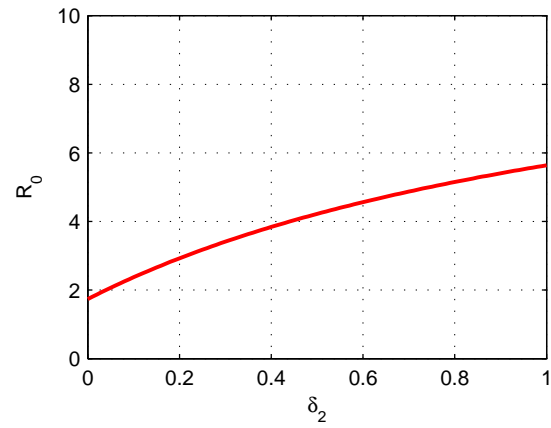

(d) $R_{0}$ as a function of $\delta_{2}$

Figure 2: Sensitivity of parameters

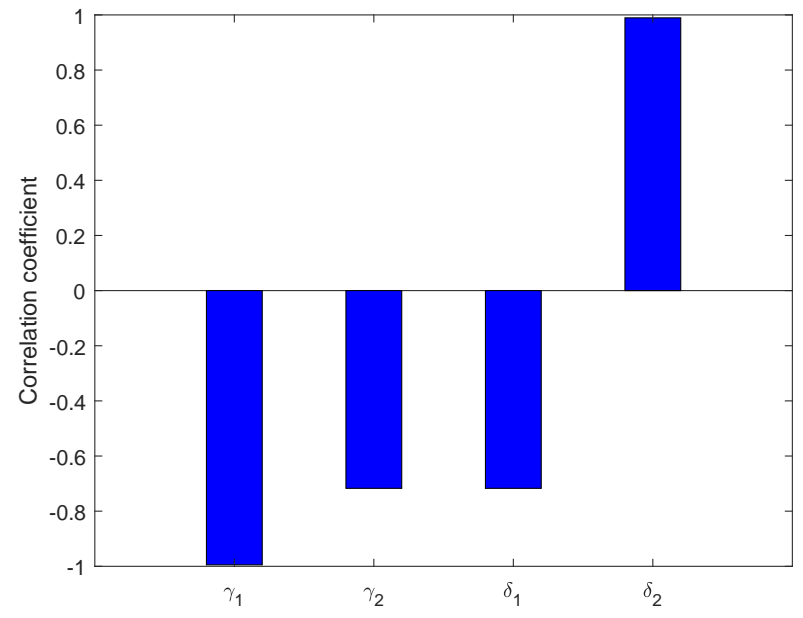

Figure 3: Correlation coefficients 


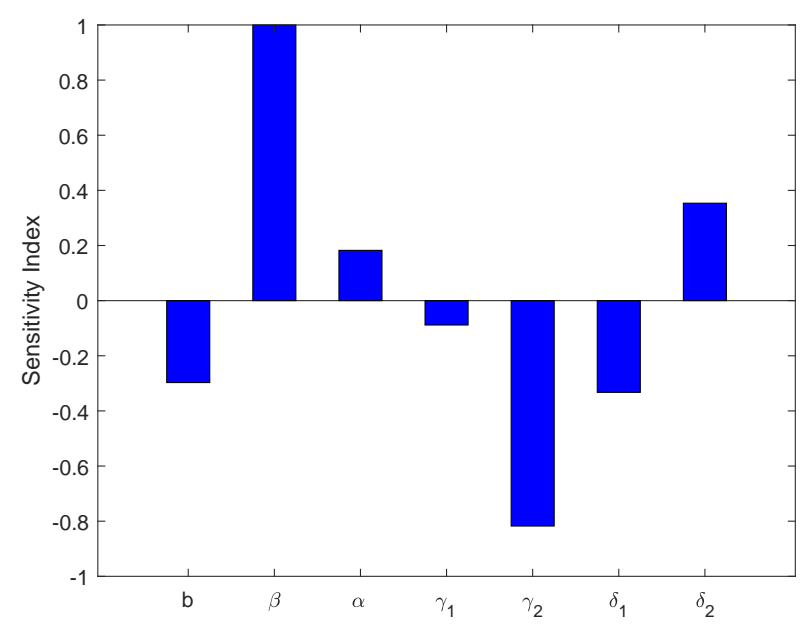

Figure 4: Sensitivity index

can lead to the increase of $R_{0}$. Fig. 3 shows that the correlations of $\gamma_{1}, \gamma_{2}, \delta_{1}, \delta_{2}$ and $R_{0}$ are significant, and only the correlation of $\delta_{2}$ and $R_{0}$ is positive. Therefore, the correlations may be influenced by the parameter values. Following the definition of the sensitivity index $[33,34]$, if $x$ is a parameter, the sensitivity index of $R_{0}$ with respect to $x$ can be written as

$$
\Gamma_{x}^{R_{0}}=\frac{\partial R_{0}}{\partial x} \times \frac{x}{R_{0}}
$$

Fig. 4 shows the sensitivity index of $R_{0}$ with respect to the parameters. The results show that the role of incomplete recovery is less than that of relapse.

According to Theorems 3.4 and 3.5, Fig. 5 illustrates the global stability of model (2.3) by time series diagram with different initial values. To show the advantages of the optimal vaccination control, five constant controls (the control degrees are $v=0$, $v=0.25, v=0.5, v=0.75, v=1$ respectively) and optimal control are compared in Fig. 6. The left column of Fig. 6 shows that the optimal vaccination control restrains the infected density as well as the maximal control $(v=1)$, but the cost of it is less than the maximal control. From Eq. (4.1) and (4.2), the cost includes cost of infected people and cost of vaccination. The last picture of Fig. 6 shows the difference of cost of vaccination between the maximal control and the optimal control.

The parameter $B_{k}$ is a ratio. The value of $B_{k}$ of Fig. 6 is 0.05 , it represents that for one person the cost of vaccination is 5 percent of that of treatment. It is only 


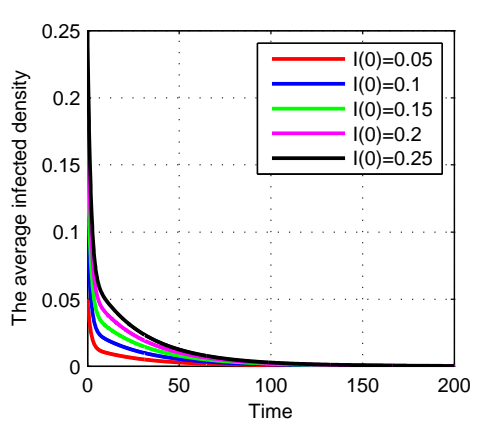

(a) $R_{0}=0.5852$

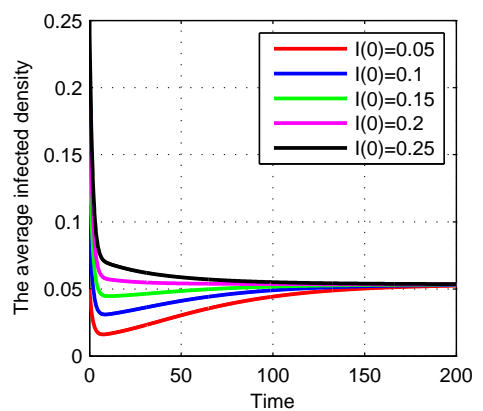

(c) $R_{0}=2.9258$

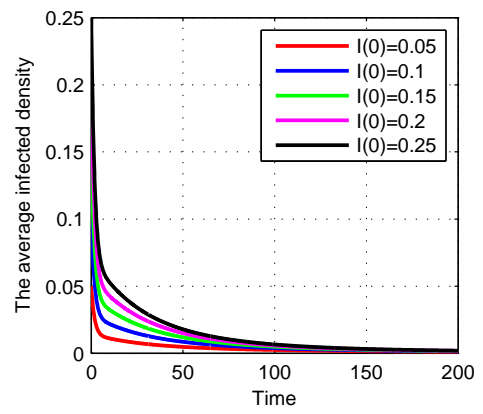

(b) $R_{0}=1$

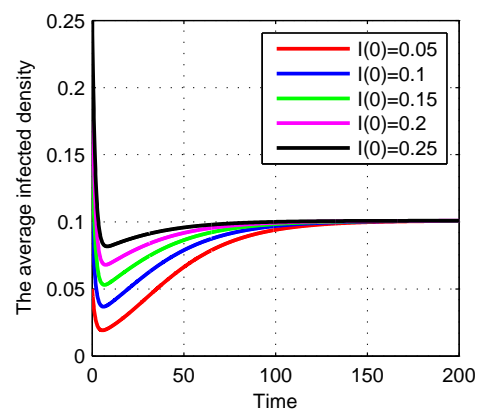

(d) $R_{0}=4.3888$

Figure 5: Global stability of the model

an assumption. Changing the value of $B_{k}$, Fig. 7 shows similar results as those of Fig. 6. The difference is that the total cost increases with the increase of $B_{k}$. And the difference in cost also increases with the increase of $B_{k}$. In addition, different value of $B_{k}$ leads to different optimal control. To see the optimal control degree in details, control degree of each node is shown in Fig. 8. Consistent with Fig. 6 and Fig. 7, Fig. 8 shows that control degree decreases as times go on. When $B_{k}=0.05$, for each node, the control degree is different. The control degree of nodes with small degree and nodes with big degree are both strong. When $B_{k}=0.1$, the result is similar to that of the case of $B_{k}=0.05$, but when $n>200$ the result is complicated.

\section{Conclusion and Discussion}

In this paper, an SEIRS model with incomplete recovery on scale-free networks is proposed. We analyzed the global behavior of the model. $R_{0}$ is obtained. In addition, when $R_{0} \leq 1$, the disease-free equilibrium $E_{0}$ is globally stable; when $R_{0}>1$, the 

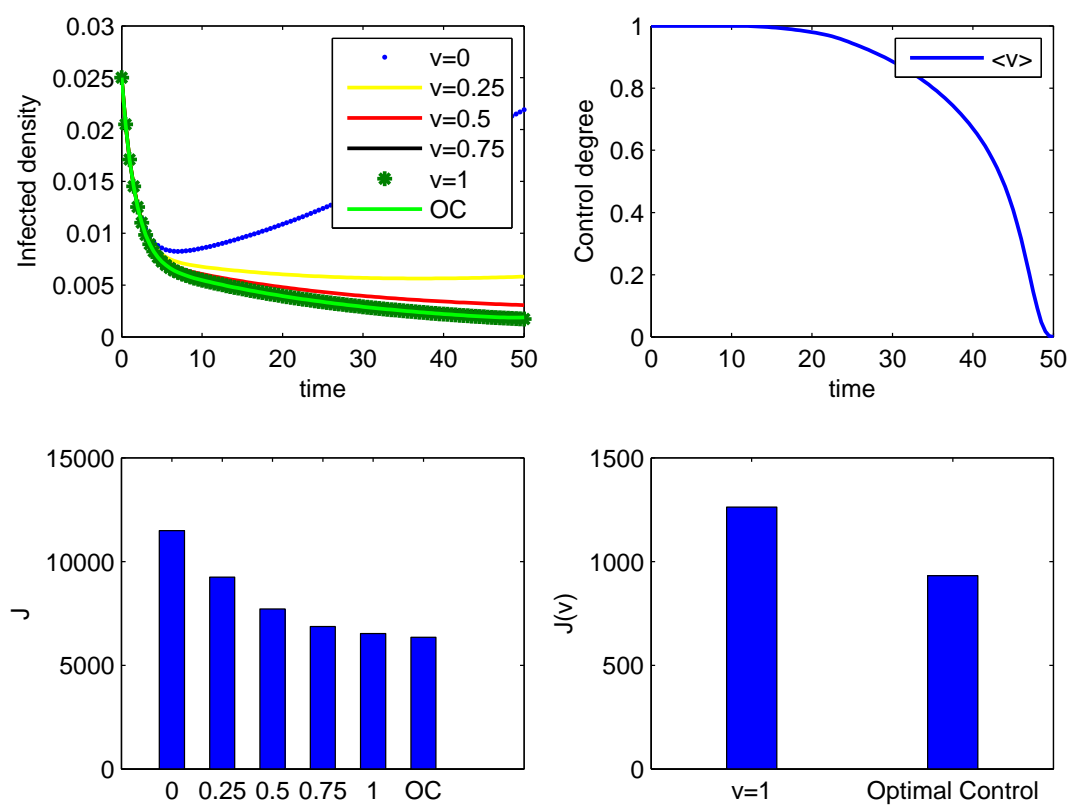

Figure 6: Sensitivity of control strategies
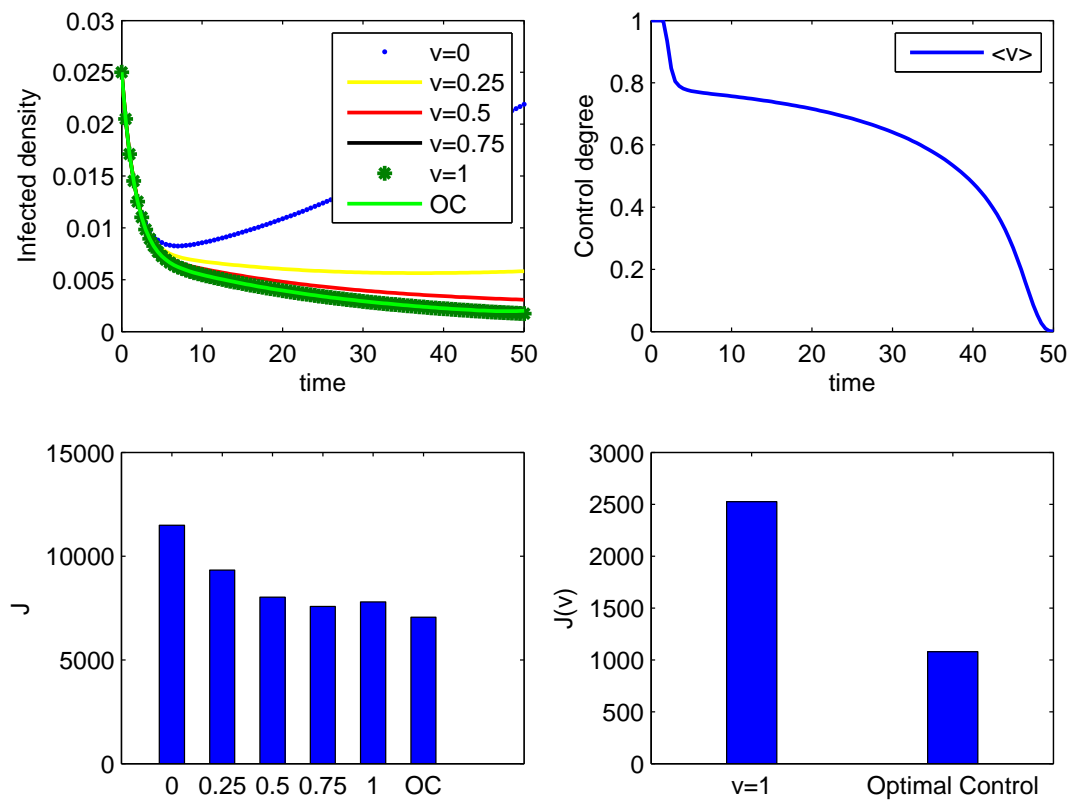

Figure 7: Sensitivity of control strategies 


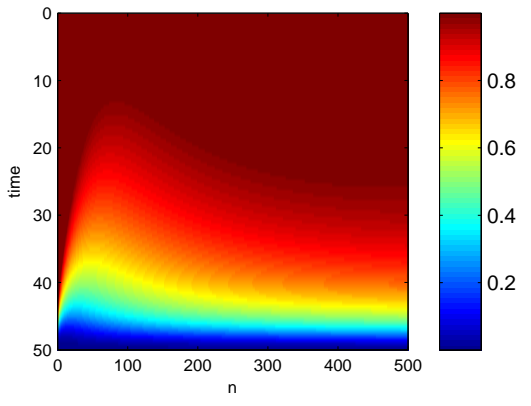

(a) $B_{k}=0.05$

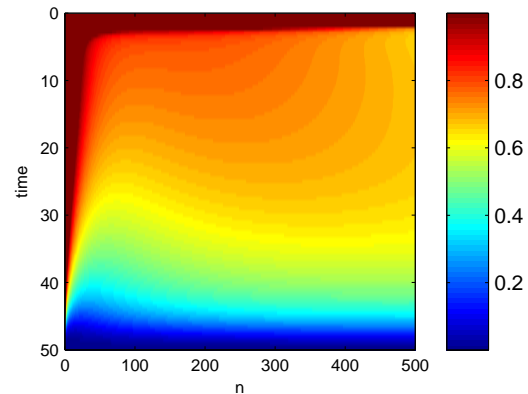

(b) $B_{k}=0.1$

Figure 8: Control degree of each node

positive equilibrium is globally stable. Then time-varying vaccination control of the model is established, and the existence and the solution of the optimal control are solved. At last, numerical simulation is presented to illustrate the theoretical results. The numerical simulation shows that optimal control can restrain the infected density as well as the maximal control but the cost is less. And the optimal control degree varies as the ratio of vaccination cost to treatment cost varies.

In our model, appropriate vaccination can reduce the infected density to a very low level but can not clear up the infection. The reason may be that incomplete recovery and relapse may lead to complicated situation. In our model, the study is based on the assumption that incomplete recovery and relapse are independent. In fact, incomplete recovery can lead to relapse. There is no detailed information about the relationship between incomplete recovery and relapse in existing studies. Thus the relevant research is expected.

Herpes and tuberculosis are infectious diseases spread all over the world. Due to incomplete recovery and relapse, they cannot be cleared up despite of the high degree of attention. The efforts of medical workers are expected to remove incomplete recovery and relapse. And we hope that our research will have practical value in controlling the spread of similar diseases. 


\section{Acknowledgements}

The authors would like to express their heartfelt appreciation for the financial support under the outstanding youth fund of North University of China. Moreover, they are grateful to the anonymous reviewers.

\section{Availability of data and materials}

The datasets generated during and analysed during the current study are available from the corresponding author on reasonable request.

\section{Conflict of interest}

The authors declare that they have no conflict of interest.

\section{References}

[1] D. Tudor, A deterministic model for herpes infections in human and animal populations, SIAM Rev. 32 (1990) 136-139.

[2] K. Styblo, Selected Papers: Epidemiology of Tuberculosis, Royal Netherlands Tuberculosis Association 24 (1991) 5-62.

[3] P. G. Smith, A. R. Moss, Epidemiology of tuberculosis, Bloom B. R. (ed.) Tuberculosis: Pathogenesis, protection, and control (1994) 47-59.

[4] H.N. Moreira, Y. Wang, Global stability in a $\mathrm{S} \rightarrow \mathrm{I} \rightarrow \mathrm{R} \rightarrow \mathrm{I}$ model, SIAM Rev. 39 (1997) 497-502.

[5] C. Castillo-Chavez, Z. Feng, To treat of not to treat: the case of tuberculosis, J. Math. Biol. 35 (1997) 629-656.

[6] P. van den Driessche, J. Watmough, Reproduction numbers and sub-threshold endemic equilibria for compartmental models of disease transmission, Math. Biosci. 180 (2002) 29-48. 
[7] P. van den Driessche, X. Zou, Modeling relapse in infectious diseases, Math. Biosci. 207 (2007) 89-103.

[8] P. van den Driessche, L. Wang, X. Zou, Modelling disease with latency and relapse, Math. Biosci. Eng. 4 (2007) 205-219.

[9] S. Liu, S. Wang, L. Wang, Global dynamics of delay epidemic models with nonlinear incidence rate and relapse, Nonlinear Anal-Real 12 (2011) 119-127.

[10] P. Georgescu, H. Zhang, A Lyapunov functional for a SIRI model with nonlinear incidence of infection and relapse, Appl. Math. Comput. 219 (2013) 8496-8507.

[11] Y. Chen, J. Li, S. Zou, Global dynamics of an epidemic model with relapse and nonlinear incidence, Math. Meth. Appl. Sci. 42 (2018) 1-9.

[12] X. Feng, Z. Teng, F. Zhang, Global dynamics of a general class of multi-group models with latency and relapse, Math. Biosci. Eng. 12 99-115.

[13] J. Wang, H. Shu, Global analysis on a class of multi-group SEIR model with latency and relapse, Math. Biosci. Eng. 13 (2016) 209-225.

[14] R. Pastor-Satorras, A. Vespignani, Epidemic Spreading in Scale-Free Networks , Phys. Rev. Lett. 86 (2001) 3200-3203.

[15] R. Pastor-Satorras , C. Castellano , P. Van Mieghem, A. Vespignani, Epidemic processes in complex networks, Rev. Mod. Phys. 87 (2015) 925-979.

[16] H. Huo, P. Yang, H. Xiang, Dynamics for an SIRS epidemic model with infection age and relapse on a scale-free network, J. Franklin Inst. 356 (2019) 7411-7443.

[17] A. Lahrouz, A. Settati, H. Mahjour, M. Jarroudi, M. Fatini, Global dynamics of an epidemic model with incomplete recovery in a complex network, J. Franklin Inst. 357 (2020) 4414-4436.

[18] R. M. Anderson, R. M. May, Directly transmitted infectious diseases: control by vaccination, Science 215 (1982) 1053-1060. 
[19] R. E. Mickens, A discrete-time model for the spread of periodic diseases without immunity, Biosystems 26 (1992) 193-198.

[20] L. S. Pontryagin, V. G. Boltyanskii, R. V. Gamkrelidze, E. F. Mishechenko, The Mathematical Theory of Optimum Processes, Wiley, New York 1962.

[21] W. H. Fleming, R. W. Rishel, Deterministic and stochastic optimal control. New York: Springer-Verlag, 1975.

[22] J. Müller, Optimal vaccination patterns in age-structured populations, SIAM J. Appl. Math. 59 (1998) 222-241.

[23] G. Zaman, Y. H. Kang, I. H. Jung, Stability analysis and optimal vaccination of an SIR epidemic model, Biosystems 93 (2008) 240-249.

[24] G. Zaman, Y. H. Kang, G. Cho, I. H. Jung, Optimal strategy of vaccination and treatment in an SIR epidemic model, Math. Comput. Simul. 136 (2017) 63-77.

[25] L. J. Chen, J. T. Sun, Global stability and optimal control of an SIRS epidemic model on heterogeneous networks, Physica A 410 (2014) 196-204.

[26] L. J. Chen, J. T. Sun, Optimal vaccination and treatment of an epidemic network model, Phys. Lett. A 378 (2014) 3028-3036.

[27] K. Li, G. Zhu, Z. Ma, L. Chen, Dynamic stability of an SIQS epidemic network and its optimal control, Commun. Nonlinear Sci. 66 (2019) 84-95.

[28] L. Zhang, M. Liu, B. Xie, Optimal control of an SIQRS epidemic model with three measures on networks, Nonlinear Dyn. 103 (2021) 2097-2107.

[29] J. Liu, T. Zhang, Epidemic spreading of an SEIRS model in scale-free networks, Commun Nonlinear Sci Numer Simulat 16 (2011) 3375-3384.

[30] J. LaSalle, The stability of dynamical systems, in: Regional Conference Series in Applied Mathematics, SIAM, Philadelphia, 1976.

[31] G. Liu, Z. Liu, Z. Jin, Dynamics analysis of epidemic and information spreading in overlay networks, J. Theor. Biol. 444 (2018) 28-37. 
[32] X-Q. Zhao, Z.J. Jing, Global asymptotic behavior in some cooperative systems of functional differential equations, Can. Appl. Math. Q. 4 (1996) 421-444.

[33] T.K. Kar, S.K. Nandi, S. Jana, M. Mandal, Stability and bifurcation analysis of an epidemic model with the effect of media, Chaos Solitons Fractals 120 (2019) 188-99.

[34] M. Mandal, S. Jana, S. K. Nandi, A. Khatua, S. Adak, T.K. Kar, A model based study on the dynamics of COVID-19: Prediction and control, Chaos Solitons Fractals 136 (2020) 10988. 
Figures

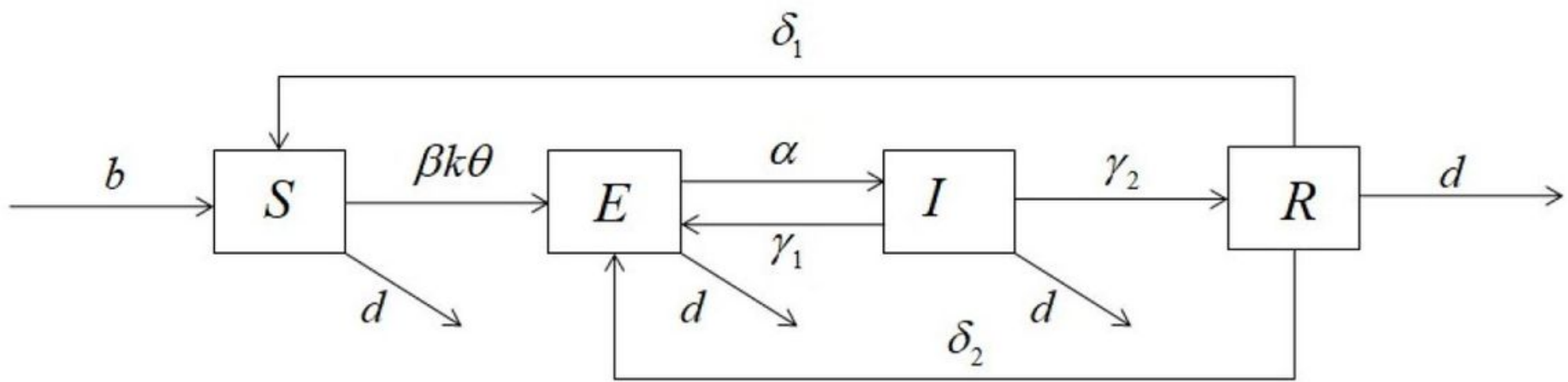

Figure 1

The disease transmission flow

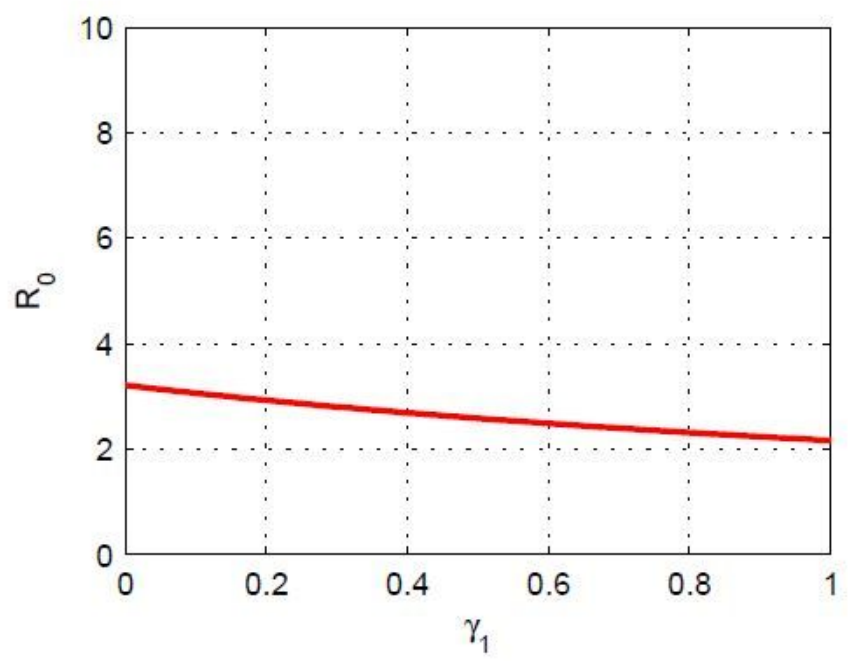

(a) $R_{0}$ as a function of $\gamma_{1}$

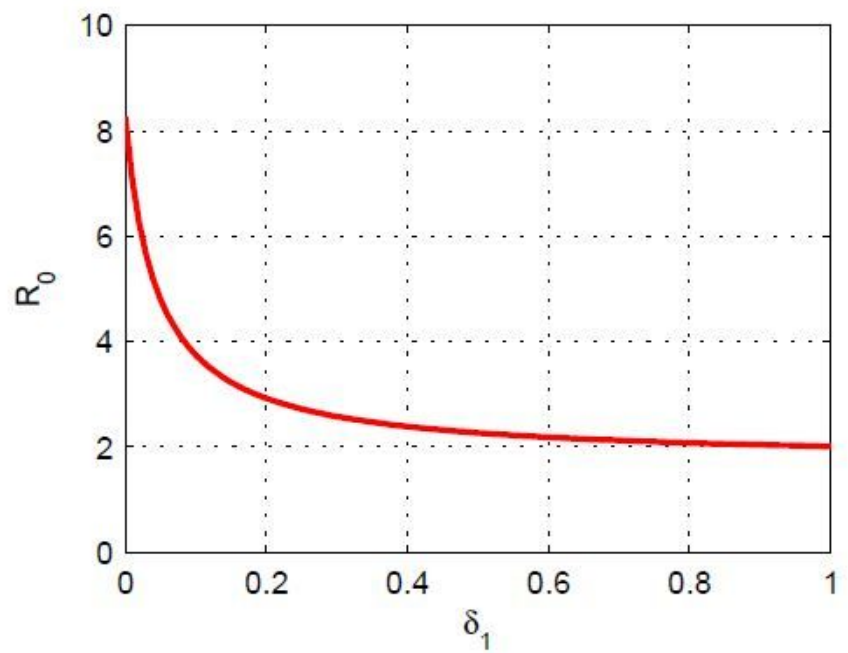

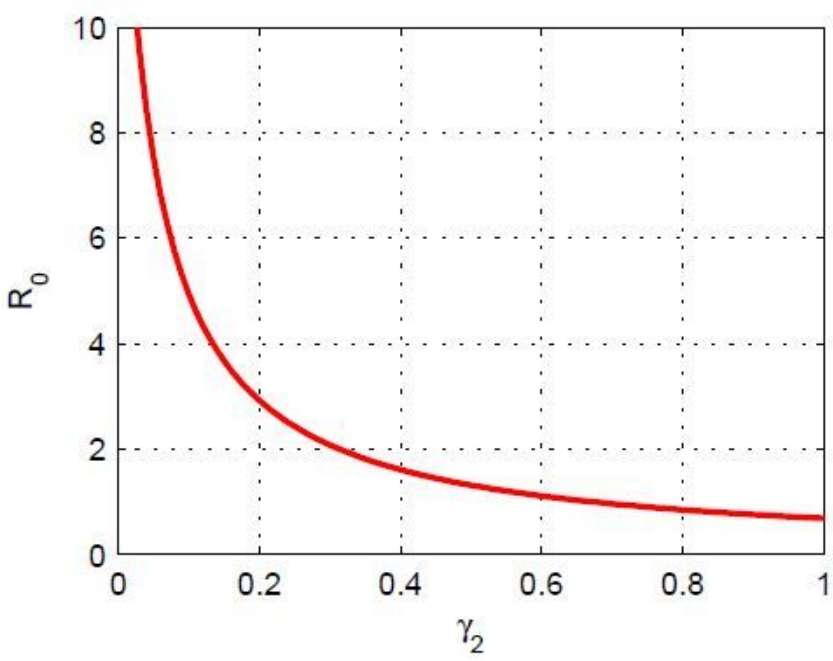

(b) $R_{0}$ as a function of $\gamma_{2}$

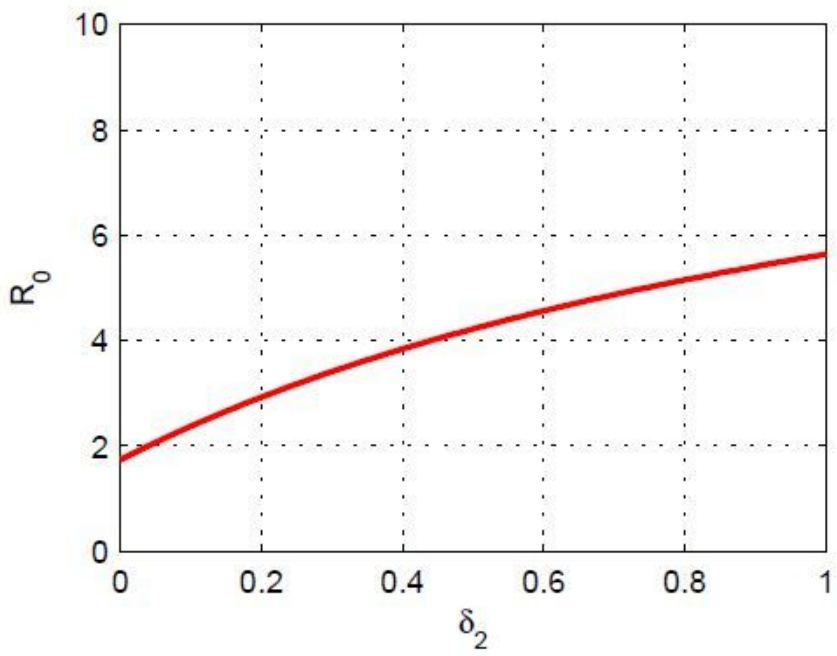


Figure 2

Sensitivity of parameters

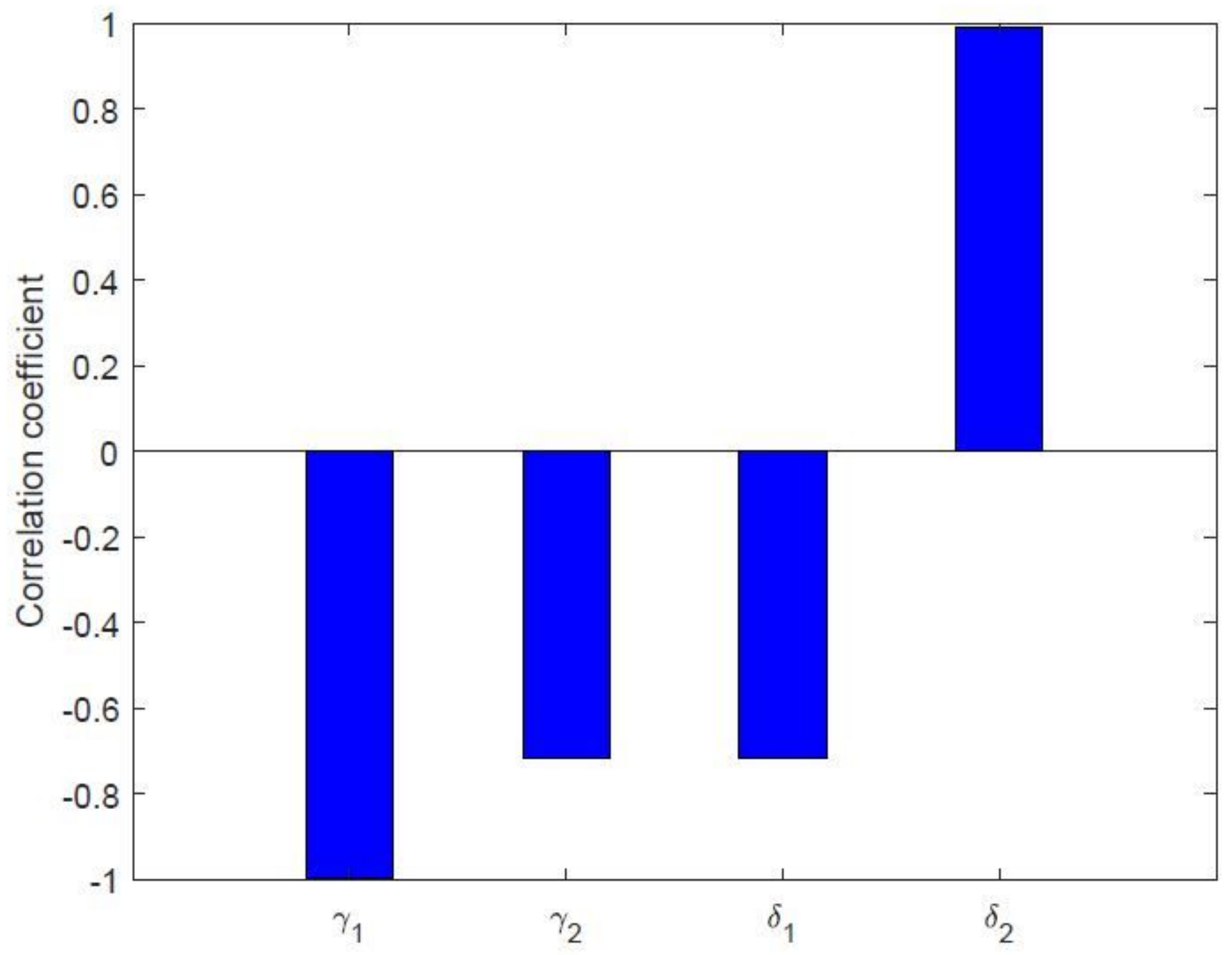

Figure 3

Correlation coefficients 


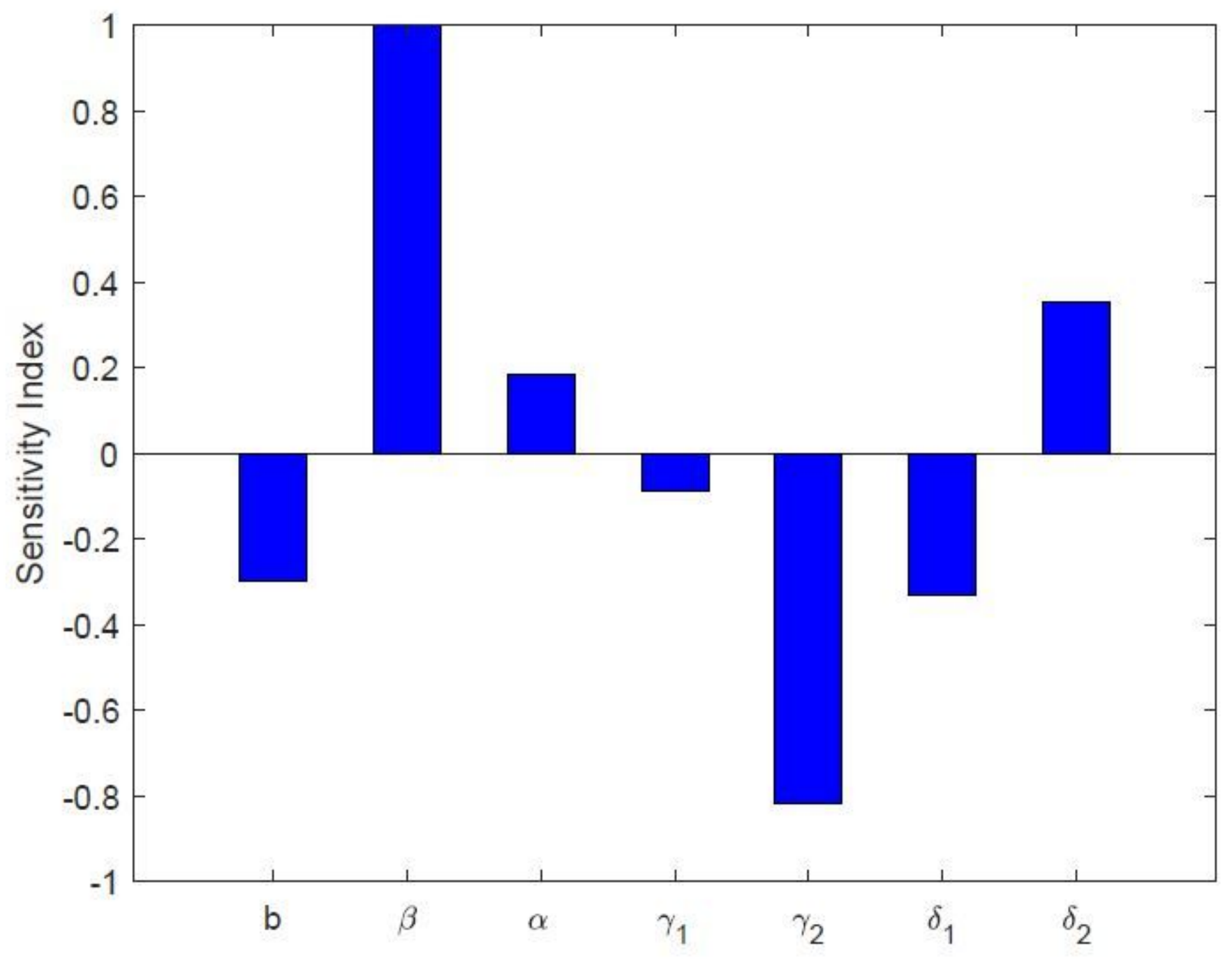

Figure 4

Sensitivity index 


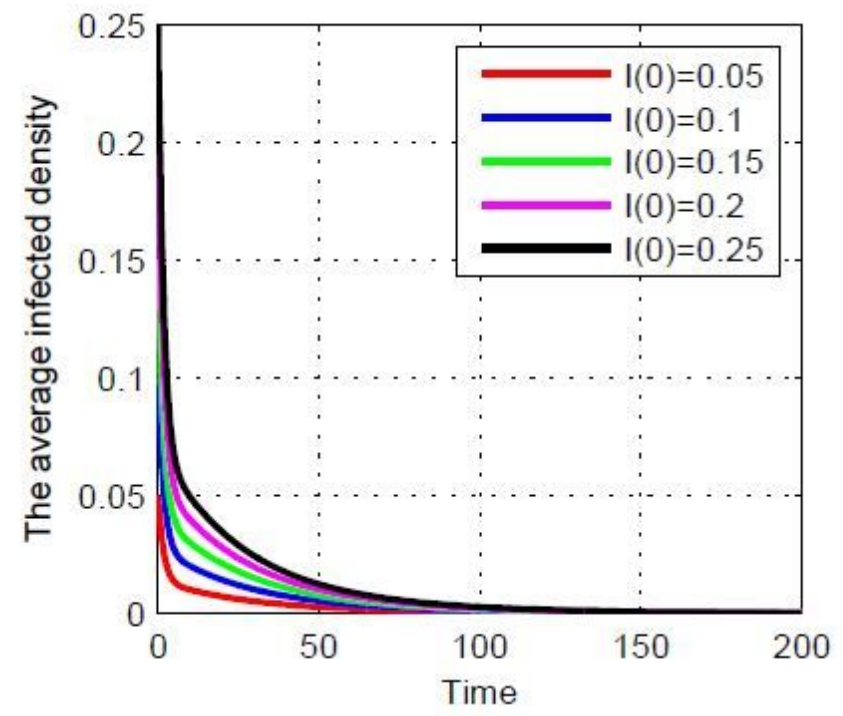

(a) $R_{0}=0.5852$

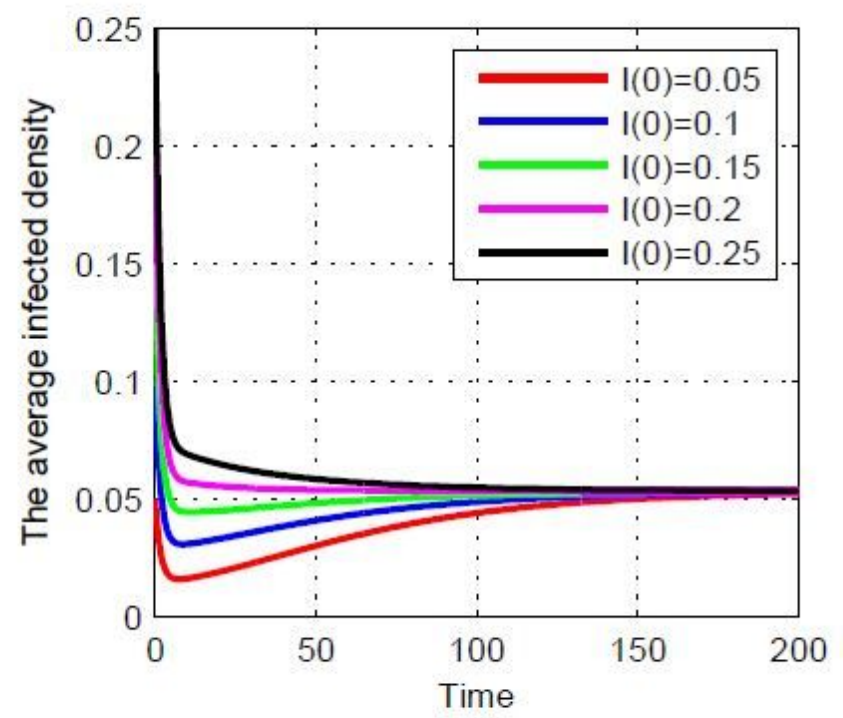

(c) $R_{0}=2.9258$

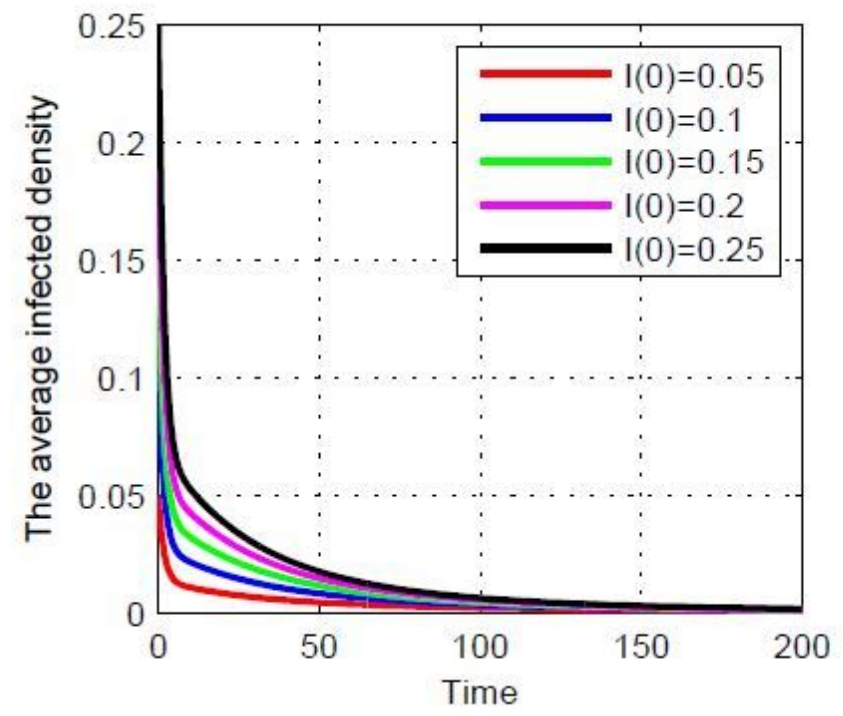

(b) $R_{0}=1$

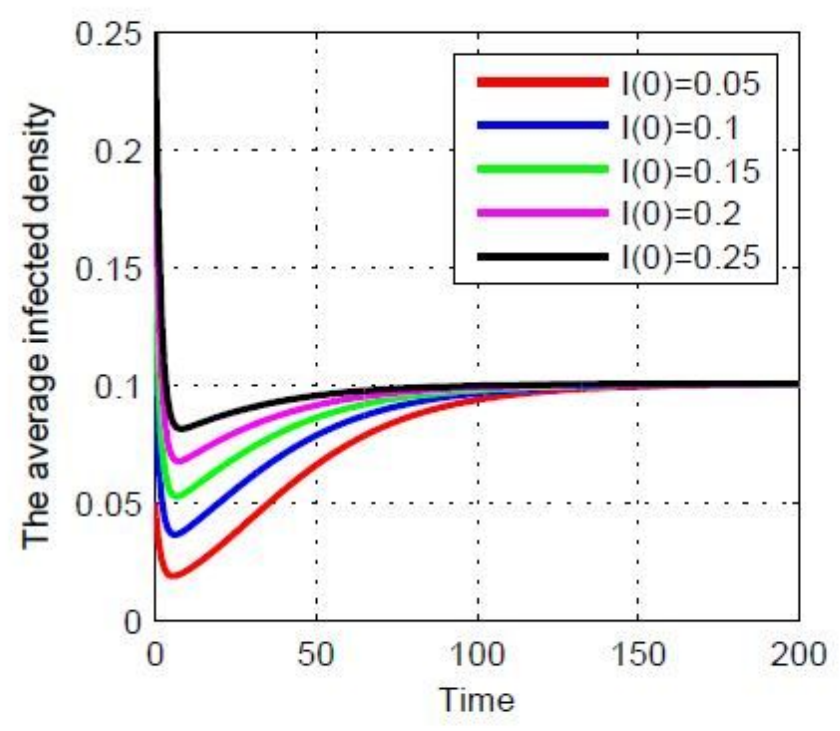

(d) $R_{0}=4.3888$

Figure 5

Global stability of the model 

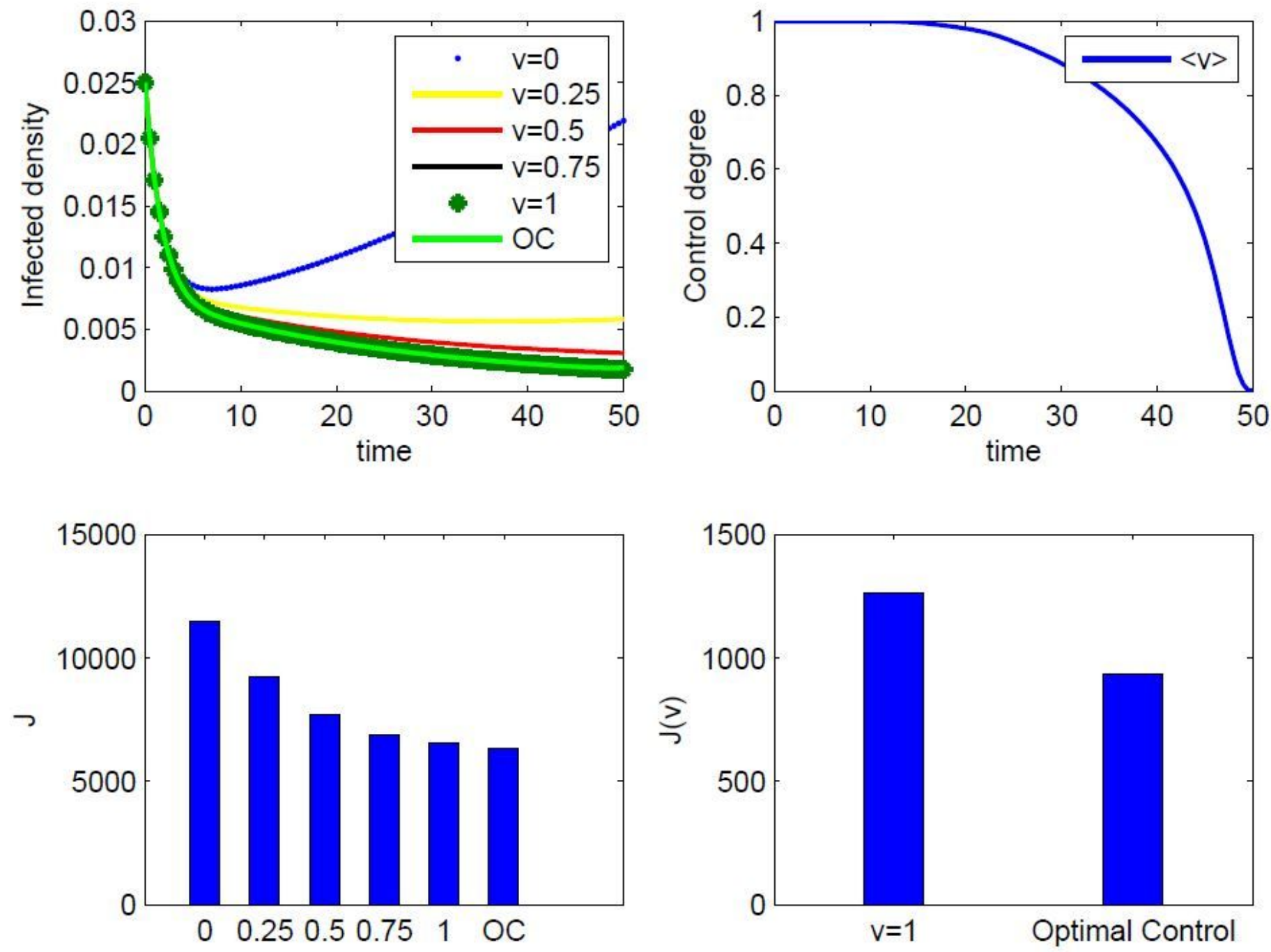

Figure 6

Sensitivity of control strategies 

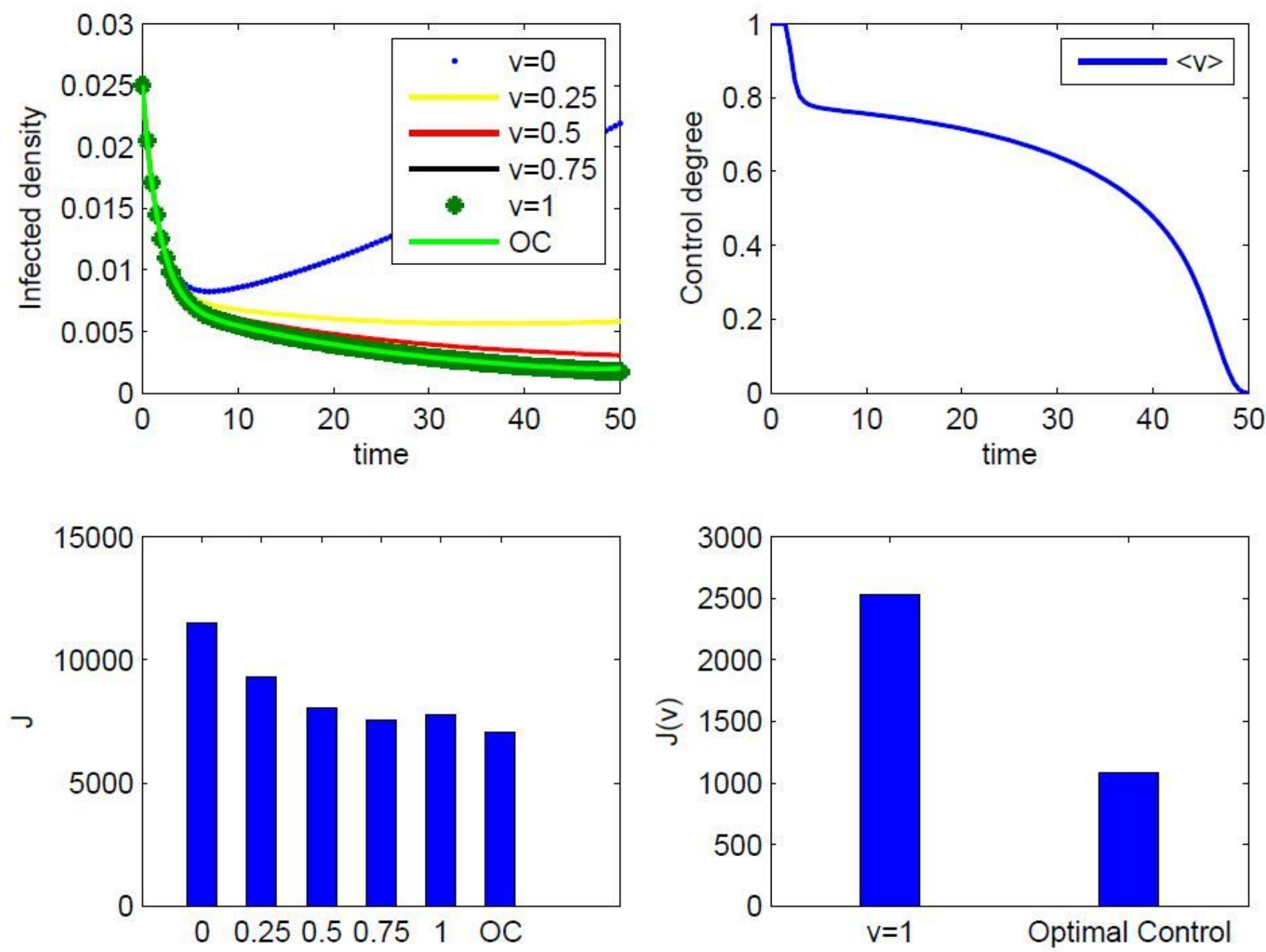

Figure 7

Sensitivity of control strategies 


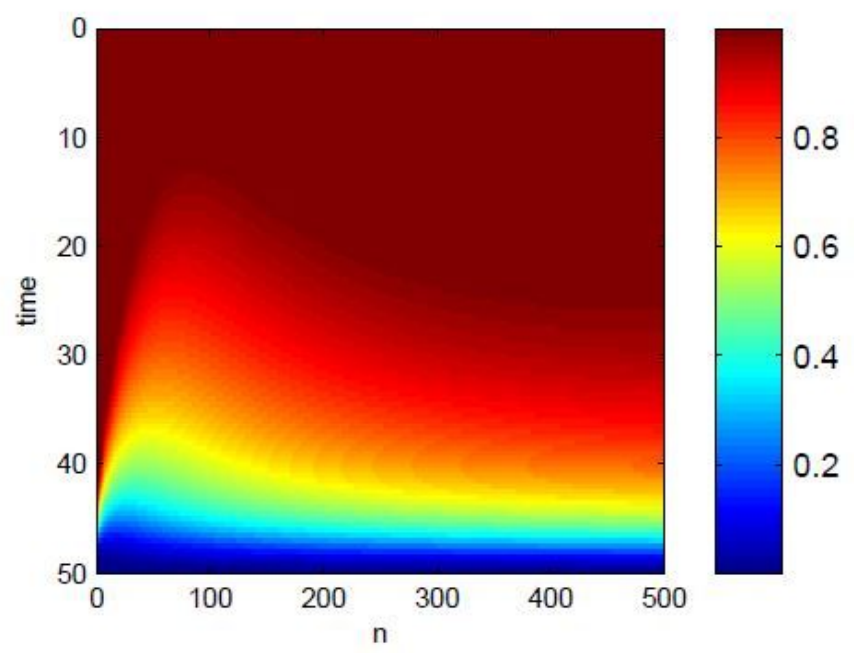

(a) $B_{k}=0.05$

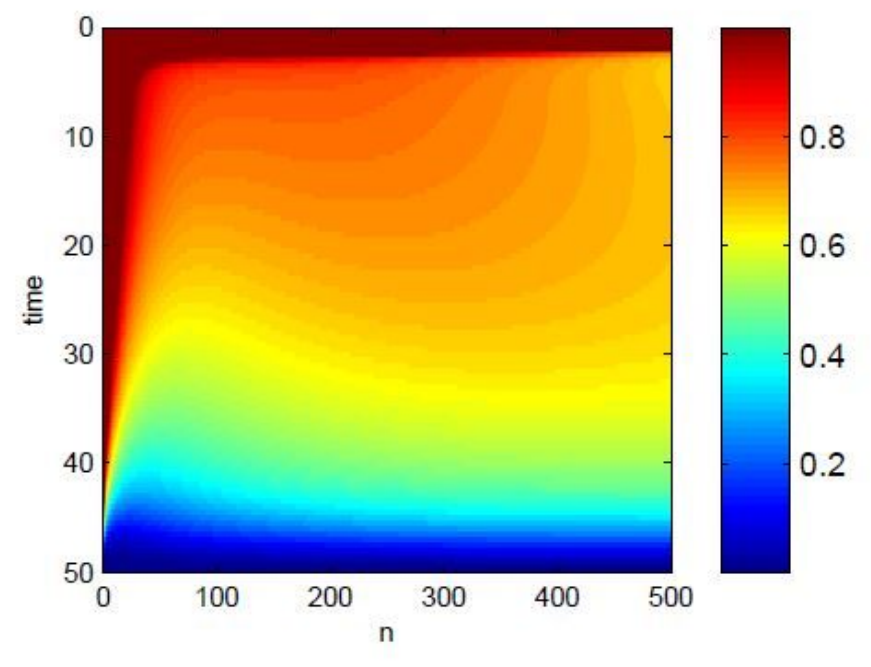

(b) $B_{k}=0.1$

Figure 8

Control degree of each node 